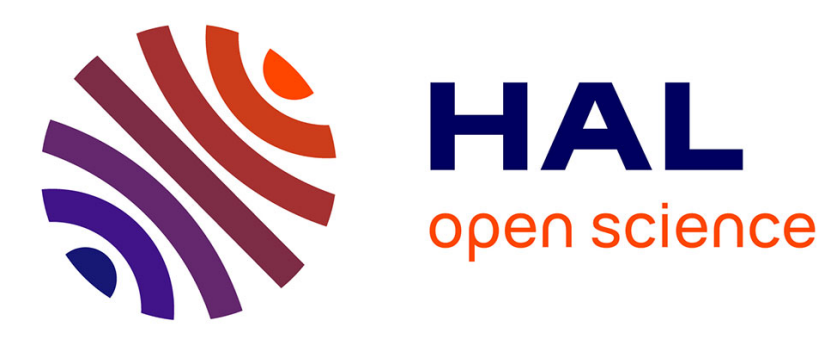

\title{
The zero relaxation limit for the Aw-Rascle-Zhang traffic flow model
}

Paola Goatin, Nicolas Laurent-Brouty

\section{To cite this version:}

Paola Goatin, Nicolas Laurent-Brouty. The zero relaxation limit for the Aw-Rascle-Zhang traffic flow model. Zeitschrift für Angewandte Mathematik und Physik, 2019, 70 (31), 10.1007/s00033-018-10711. hal-01760930v3

\section{HAL Id: hal-01760930 \\ https://hal.inria.fr/hal-01760930v3}

Submitted on 11 Apr 2019

HAL is a multi-disciplinary open access archive for the deposit and dissemination of scientific research documents, whether they are published or not. The documents may come from teaching and research institutions in France or abroad, or from public or private research centers.
L'archive ouverte pluridisciplinaire HAL, est destinée au dépôt et à la diffusion de documents scientifiques de niveau recherche, publiés ou non, émanant des établissements d'enseignement et de recherche français ou étrangers, des laboratoires publics ou privés. 


\title{
The zero relaxation limit for the Aw-Rascle-Zhang traffic flow model
}

\author{
Paola Goatin* $\quad$ Nicolas Laurent-Brouty ${ }^{\dagger}$
}

January 1, 2019

\begin{abstract}
We study the behavior of the Aw-Rascle-Zhang model when the relaxation parameter converges to zero. In a Lagrangian setting, we use the Wave-Front-Tracking method with splitting technique to construct a sequence of approximate solutions. We prove that this sequence converges to a weak entropy solution of the relaxed system associated to a given initial datum with bounded variation. Besides, we also provide an estimate on the decay of positive waves. We finally prove that the solutions of the Aw-Rascle-Zhang system with relaxation converge to a weak solution of the corresponding scalar conservation law when the relaxation parameter goes to zero.
\end{abstract}

\section{Introduction}

Conservations laws have first been used to model traffic flow as a fluid in mid-fifties by Lighthill and Whitham [33] and Richards [38]. This became the Lighthill-Whitham-Richards (LWR) model, which consists in a single conservation law accounting for the conservation of cars:

$$
\partial_{t} \rho+\partial_{x}(\rho v(\rho))=0, \quad x \in \mathbb{R}, t>0,
$$

where $\rho=\rho(t, x)$ represents the density of vehicles on the road and $v=v(\rho)$ denotes the mean velocity of the flow. In order to capture particular characteristics of traffic flow, like stop-and-go waves, second-order models were developed afterwards. The first one was the Payne-Whitham model (PW) [36, 42. Unfortunately, as mentioned in [21], it presents some drawbacks, like the unrealistic fact that information may propagate faster than the actual velocity of cars. Taking these limitations into account, Aw, Rascle [5] and Zhang [43], proposed a new two-equations model, which is referred to as the Aw-Rascle-Zhang (ARZ) model:

$$
\left\{\begin{array}{l}
\partial_{t} \rho+\partial_{x}(\rho v)=0, \\
\partial_{t}(v+p(\rho))+v \partial_{x}(v+p(\rho))=0,
\end{array} \quad x \in \mathbb{R}, t>0,\right.
$$

\footnotetext{
${ }^{1}$ Paola Goatin

Inria Sophia Antipolis - Méditerranée, Université Côte d'Azur, Inria, CNRS, LJAD, 2004 route des Lucioles BP 93, 06902 Sophia Antipolis Cedex, France.

E-mail: paola.goatin@inria.fr ORCID: 0000-0001-5169-1751

${ }^{2}$ Nicolas Laurent-Brouty (corresponding author) Université Côte d'Azur, Inria, CNRS, LJAD, France. Ecole des Ponts ParisTech, Champs-sur-Marne, France. 2004, route des Lucioles - BP 93, 06902 Sophia Antipolis Cedex, France. E-mail: nicolas.laurent-brouty@inria.fr phone: +33497155311 ORCID: 0000-0001-6574-4367
} 
where $p(\rho)$ is a pseudo-pressure function accounting for drivers' anticipation of downstream density changes. To ensure that system 1.2 is strictly hyperbolic, with a genuinely nonlinear and a linearly degenerate characteristic fields, we impose the following constraints:

$$
\rho>0, \quad p(\rho)>0, \quad p^{\prime}(\rho)>0, \quad 2 p^{\prime}(\rho)+\rho p^{\prime \prime}(\rho)>0 .
$$

Subsequently, in [37] and [23] the authors suggest to add a relaxation term, to ensure that the velocity of the flow is relaxed towards the equilibrium speed:

$$
\left\{\begin{array}{l}
\partial_{t} \rho+\partial_{x}(\rho v)=0 \\
\partial_{t}(v+p(\rho))+v \partial_{x}(v+p(\rho))=\frac{V(\rho)-v}{\delta},
\end{array}\right.
$$

where $V(\rho) \geqslant 0$ is a non-increasing function which represents the equilibrium speed, and $\delta>0$ is a relaxation parameter. To ensure a well-defined problem, the system must satisfy the so-called sub-characteristic condition [14, 15] :

$$
-p^{\prime}(\rho) \leqslant V^{\prime}(\rho) \leqslant 0 \quad \text { for } \rho>0 .
$$

Note that if $p^{\prime}(\rho)=-V^{\prime}(\rho)$, the system can be decoupled and reduced to the scalar case 31. The case $V^{\prime}(\rho)=0$ does not make sense for traffic modeling, since it would imply that the equilibrium velocity is independent of the density. For these reasons, we will consider strict inequalities in the rest of the paper:

$$
-p^{\prime}(\rho)<V^{\prime}(\rho)<0 \quad \text { for } \rho>0 .
$$

Multiplying the first equation of $(1.4)$ by $p^{\prime}(\rho)$ and subtracting its terms to the second equation, we obtain:

$$
\left\{\begin{array}{l}
\partial_{t} \rho+\partial_{x}(\rho v)=0 \\
\partial_{t} v+\left(v-\rho p^{\prime}(\rho)\right) \partial_{x} v=\frac{V(\rho)-v}{\delta}
\end{array}\right.
$$

Under hypotheses (1.3), the associated homogeneous system is strictly hyperbolic (away from vacuum) with eigenvalues $\lambda_{1}=v-\rho p^{\prime}(\rho)$ and $\lambda_{2}=v$.

The conservative form of (1.4), 1.7] is given by (see [5]):

$$
\left\{\begin{array}{l}
\partial_{t} \rho+\partial_{x}(\rho v)=0 \\
\partial_{t}(\rho(v+p(\rho)))+\partial_{x}(\rho v(v+p(\rho)))=\rho \frac{V(\rho)-v}{\delta} .
\end{array}\right.
$$

Defining $w:=v+p(\rho)$, we obtain:

$$
\left\{\begin{array}{l}
\partial_{t} \rho+\partial_{x}(\rho v)=0 \\
\partial_{t}(\rho w)+\partial_{x}(\rho v w)=\rho \frac{V(\rho)-v}{\delta}
\end{array}\right.
$$

which can be rewritten into Lagrangian coordinates $(t, X)$ [18] as follows. We denote by $\tau$ the specific volume, such that $\tau=\frac{1}{\rho}$. Using the notations

$$
\tilde{p}(\tau)=p\left(\frac{1}{\tau}\right), \quad \tilde{V}(\tau)=V\left(\frac{1}{\tau}\right), \quad \tilde{p}^{\prime}(\tau)=-\frac{1}{\tau^{2}} p^{\prime}\left(\frac{1}{\tau}\right)<0,
$$

we obtain the following equivalent hyperbolic system

$$
\left\{\begin{array}{l}
\partial_{t} \tau-\partial_{X} v=0 \\
\partial_{t} w=\frac{\tilde{V}(\tau)-v}{\delta}
\end{array}\right.
$$


where

$$
\partial_{x} X=\rho, \quad \partial_{t} X=-\rho v
$$

with initial data

$$
\left\{\begin{array}{l}
\tau(0, \cdot)=\tau_{0}, \\
w(0, \cdot)=w_{0} .
\end{array}\right.
$$

Remark. We remind the reader that systems $(1.8)$ and $(1.9)$ are equivalent if we consider classical solutions, but weak solutions must be treated more carefully, particularly in the presence of vacuum. We refer to [41] for precise equivalence conditions between weak solutions of Eulerian and Lagrangian systems.

In Lagrangian notations, the sub-characteristic condition $(1.6)$ becomes

$$
\tilde{p}^{\prime}(\tau)<-\tilde{V}^{\prime}(\tau)<0
$$

The homogeneous system in (1.9) admits as eigenvalues $\lambda_{1}=\tilde{p}^{\prime}(\tau)=-\rho^{2} p^{\prime}(\rho)<0$ and $\lambda_{2}=0$, and the associated Riemann invariants are $w$ and $v$. The first characteristic field is genuinely nonlinear, while the second is linearly degenerate. The system is a Temple class system, since shock and rarefaction curves coincide [40].

The literature about relaxation limits of hyperbolic systems of conservation laws is huge, starting from the seminal paper of Chen, Levermore and Liu [14], which is based on the compensated compactness method. Results relying on the Wave-Front Tracking (WFT) method [20, 25] were obtained in 2 for the $2 \times 2$ elasticity system and in [1 for a $3 \times 3$ system modeling multiphase reactive flows. For works concerning traffic models, we recall the result by Lattanzio and Marcati [27] on the Payne-Whitham model with relaxation, which proves the convergence to the equilibrium solutions for $L^{\infty}$ initial data by compensated compactness. Several results by Li [28, 29, 30, 31, 32] concern either different models, or an ARZ-type model in which the pressure $p$ satisfies $p^{\prime}(\rho)=-V^{\prime}(\rho)$. They are mainly achieved using Glimm's scheme or finite difference approximations.

In this work, we provide a rigorous proof of existence of solutions for the relaxed ARZ system (1.9), as well as the convergence of these solutions to the equilibrium LWR equation

$$
\partial_{t} \tau-\partial_{X} \tilde{V}(\tau)=0,
$$

as $\delta \rightarrow 0$. The proofs are based on the construction of approximate solutions by means of the wave-front tracking technique. This choice is motivated by the lack of Total Variation $(T V)$ bounds on classical finite volume (Godunov) approximations, which were used in 4, 6, 23. Indeed, it is well-known that, since these schemes are constructed taking means of the conservative variables, they are not able to preserve the total variation of Riemann invariants. In particular, in the case of the ARZ system, they may not capture correctly contact discontinuities, and adapted schemes must be used [13. This point will be discussed in the Appendix.

The paper is organized as follows. In Section 2, we detail the construction of WFT approximations and we derive the necessary $L^{\infty}$ and $T V$ uniform bounds to guarantee their convergence towards a solution of (1.9), which is detailed in Section 3 . In Section 4 we prove some estimates for positive genuinely nonlinear waves occurring in solutions of system (1.9). Finally, Section 5 is devoted to the proof of the relaxation limit. 


\section{WFT approximations}

In this section, we construct wave-front tracking approximations of the solution following the approach developed for similar cases in [2, 7]. The approximate solution is constructed in a twostep process, which successively solves the homogeneous system for a given piecewise constant initial datum, and then integrate the source term contained in the following ODE:

$$
w_{t}=\frac{\tilde{V}(\tau)-v}{\delta} .
$$

Given two constants $0<\check{\tau}<\hat{\tau}<+\infty$, let us define the domain $\mathbf{E}$ as

$$
\begin{array}{r}
\mathbf{E}=\mathbf{E}(\check{\tau}, \hat{\tau}):=\{u=(\tau, w) \in[\check{\tau}, \hat{\tau}] \times[0,+\infty[: \tilde{V}(\check{\tau}) \leqslant w-\tilde{p}(\tau) \leqslant \tilde{V}(\hat{\tau}), \\
\tilde{V}(\hat{\tau})+\tilde{p}(\hat{\tau}) \leqslant w \leqslant \tilde{V}(\check{\tau})+\tilde{p}(\check{\tau})\} .
\end{array}
$$

In particular, note that vacuum states are excluded by the above domain. For any $M>0$, we define the family of functions:

$$
\mathcal{D}(M):=\{u: \mathbb{R} \rightarrow \mathbf{E}: T V(w(u))+T V(v(u)) \leqslant M\},
$$

where $v(u)=w-\tilde{p}(\tau)$ is the second Riemann invariant.

\subsection{Construction of approximate solutions via wave-front tracking}

Given a fixed time horizon $T>0$, consider a sequence of time-steps $\Delta t^{\nu}>0, \nu \in \mathbb{N}$, such that $\Delta t^{\nu} \underset{\nu \rightarrow \infty}{\longrightarrow} 0$. Let $U_{0}=\left(\tau_{0}, w_{0}\right) \in \mathcal{D}(M)$. For each $\nu \in \mathbb{N}$, the interval $(0, T)$ can be partitioned in segments of the form $\left[n \Delta t^{\nu},(n+1) \Delta t^{\nu}\right], n \in \mathbb{N}$. We denote $U^{\nu}(t, x)=\left(\tau^{\nu}, w^{\nu}\right)(t, x), t \in[0, T]$, $x \in \mathbb{R}$, the sequence of WFT approximations of the solution of system (1.9). We construct it iteratively, for each $\nu \in \mathbb{N}$, with the following process:

1. Define a sequence of piecewise constant functions $U_{0}^{\nu}=\left(\tau_{0}^{\nu}, w_{0}^{\nu}\right) \in \mathcal{D}(M)$ satisfying:

$$
\begin{array}{lll}
T V\left(w_{0}^{\nu}\right) \leqslant T V\left(w_{0}\right), & \left\|w_{0}^{\nu}-w_{0}\right\|_{\mathbf{L}^{\infty}} \leqslant \frac{1}{\nu}, & \left\|\tau_{0}^{\nu}-\tau_{0}\right\|_{\mathbf{L}^{1}} \leqslant \frac{1}{\nu}, \\
T V\left(v\left(U_{0}^{\nu}\right)\right) \leqslant T V\left(v\left(U_{0}\right)\right), & \left\|v\left(U_{0}^{\nu}\right)-v\left(U_{0}\right)\right\|_{\mathbf{L}^{\infty} \leqslant \frac{1}{\nu},} & \left\|v\left(U_{0}^{\nu}\right)-v\left(U_{0}\right)\right\|_{\mathbf{L}^{1}} \leqslant \frac{1}{\nu} .
\end{array}
$$

The existence of such $U_{0}^{\nu}$ is provided by [8, Lemma 2.2].

2. For each $\nu \in \mathbb{N}$, the piecewise constant function $U_{0}^{\nu}$ has a finite number of discontinuities. Solve the homogeneous system

$$
\left\{\begin{array}{l}
\partial_{t} \tau-\partial_{X} v=0 \\
\partial_{t} w=0
\end{array}\right.
$$

for each Riemann problem arising at all discontinuities for $t \in\left[0, \Delta t^{\nu}\right)$ using wave-front tracking method [7], and name $U^{\nu}(t, \cdot), t \in\left[0, \Delta t^{\nu}\right)$, the corresponding piecewise constant function.

We recall that the system $(2.3)$ admits as eigenvalues $\lambda_{1}=\tilde{p}^{\prime}(\tau)=-\rho^{2} p^{\prime}(\rho)<0$ and $\lambda_{2}=0$, and the associated Riemann invariants are $w$ and $v$. Each Riemann problem can thus be solved by a shock or rarefaction with negative speed and/or a stationary contact discontinuity. More precisely, we fix the parameter $\epsilon_{\nu}=2^{\frac{-1}{\Delta t^{\nu}}}$, and we consider 
an approximate Riemann solver in order to generate piecewise constant approximate solutions. In particular, the solver does not modify shock waves and contact discontinuities. Any rarefaction is approximated by a fan of waves of size $\epsilon_{\nu}$ moving with speed $\dot{x_{\alpha}}(t)=\lambda_{1}(\tau, w)\left(t, x_{\alpha}+\right)$ and thus violating the Rankine-Hugoniot and the entropy conditions with a global error of order $\mathcal{O}\left(\epsilon_{\nu}\right)$ (see [8, Section 7.1]). The approximate Riemann solver can then be applied at each discontinuity, and the corresponding solutions be propagated until a wave interaction occurs for $t<\Delta t^{\nu}$. We can assume without loss of generality that only two waves interact at a given time, by possibly altering slightly their speed. When two waves interact, a new Riemann problem can be solved approximately and the solution prolonged until the next interaction. 
3. At $t=\Delta t^{\nu}$, we define

$$
\begin{aligned}
\tau^{\nu}\left(\Delta t^{\nu}, \cdot\right) & =\tau^{\nu}\left(\Delta t^{\nu}-, \cdot\right) \\
w^{\nu}\left(\Delta t^{\nu}, \cdot\right) & =w^{\nu}\left(\Delta t^{\nu}-, \cdot\right)+\Delta t \frac{\tilde{V}\left(\tau^{\nu}\left(\Delta t^{\nu}, \cdot\right)\right)-v\left(U^{\nu}\left(\Delta t^{\nu}-, \cdot\right)\right)}{\delta} .
\end{aligned}
$$

Note that $\tau$ is conserved during the splitting scheme, while $w$ is updated according to the relaxation term:

$$
w^{+}=w^{-}+\Delta t \frac{\tilde{V}(\tau)-v^{-}}{\delta}
$$

see Figure 1
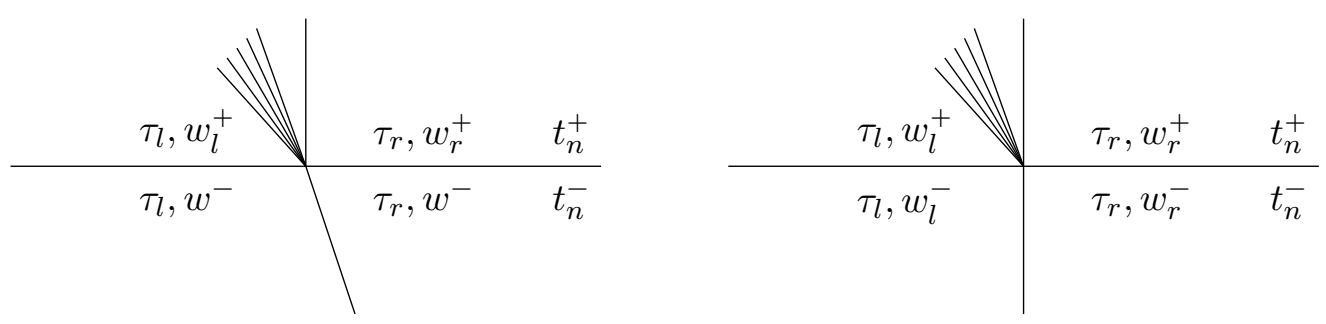

Figure 1: Notations used in step 3.

4. Treat $U^{\nu}\left(\Delta t^{\nu}, \cdot\right)$ as a new piecewise constant initial condition and repeat the previous steps 2-3 to define the solution $U^{\nu}(t, \cdot)$ for each $t \in[0, T]$, for any $T>0$ fixed.

We observe that for each $\nu$ the total number of waves generated is finite. It does not increase when solving the homogeneous system (2.3) between two consecutive time-steps, and can only increase by a finite rate at each time-step $t=t_{n}=n \Delta t^{\nu}, n \in \mathbb{N}$, when generating approximate rarefaction fans.

\subsection{Estimates on WFT approximate solutions}

In order to achieve the necessary $L^{\infty}$ uniform bounds, we identify the invariant domains [24] of system (1.9). For simplicity of notations we fix $\nu>0, \Delta t=\Delta t^{\nu}$ and work on $U=U^{\nu}$.

Lemma 2.1. For $\Delta t \leqslant \delta$, the set $\mathbf{E}$ is an invariant domain for the proposed WFT scheme.

Proof. Since $w$ and $v=w-\tilde{p}(\tau)$ are the two Riemann invariants of (2.3), which is a Temple class system, this ensures that the inequalities $\tilde{V}(\check{\tau}) \leqslant v \leqslant \tilde{V}(\hat{\tau}), \tilde{V}(\hat{\tau})+\tilde{p}(\hat{\tau}) \leqslant w \leqslant \tilde{V}(\check{\tau})+\tilde{p}(\check{\tau})$ are satisfied when solving the homogeneous system at step 2 . In addition, we know that $\tilde{p}$ is a monotone decreasing function, and that $\tau=\tilde{p}^{-1}(w-v)$. Combining this with the bounds on $v$ and $w$ we obtain that $\tau \in[\check{\tau}, \hat{\tau}]$ and then the domain $\mathbf{E}$ is invariant for the homogeneous system.

Let us now focus on the evolution step 3. Skipping the index $\nu$ for simplicity, we remind that we have:

$$
\begin{aligned}
& w^{+}=w^{-}+\frac{\Delta t}{\delta}\left(\tilde{V}(\tau)-v^{-}\right)=w^{-}\left(1-\frac{\Delta t}{\delta}\right)+\frac{\Delta t}{\delta}(\tilde{V}(\tau)+\tilde{p}(\tau)), \\
& v^{+}=w^{+}-\tilde{p}(\tau)=v^{-}\left(1-\frac{\Delta t}{\delta}\right)+\frac{\Delta t}{\delta} \tilde{V}(\tau) .
\end{aligned}
$$

Let $u^{-}=\left(\tau, w^{-}\right) \in \mathbf{E}$. The inequality $v^{+} \leqslant w^{+}$is straightforward. 
Assuming $\Delta t \leqslant \delta$, we distinguish the following situations:

- Case 1: The velocity is above the equilibrium speed

$$
v^{-} \geqslant \tilde{V}(\tau) \Leftrightarrow w^{-} \geqslant \tilde{V}(\tau)+\tilde{p}(\tau)
$$

Then

$$
\begin{aligned}
\tilde{V}(\check{\tau}) & \leqslant \tilde{V}(\tau)\left(1-\frac{\Delta t}{\delta}\right)+\frac{\Delta t}{\delta} \tilde{V}(\tau) \leqslant v^{+} \leqslant v^{-} \leqslant \tilde{V}(\hat{\tau}) \\
\tilde{V}(\hat{\tau})+\tilde{p}(\hat{\tau}) & \leqslant \tilde{V}(\tau)+\tilde{p}(\tau) \leqslant w^{+} \leqslant w^{-} \leqslant \tilde{V}(\check{\tau})+\tilde{p}(\check{\tau})
\end{aligned}
$$

therefore $u^{+}=\left(\tau, w^{+}\right) \in \mathbf{E}$.

- Case 2: The initial velocity is below the equilibrium speed

$$
v^{-} \leqslant \tilde{V}(\tau) \quad \Leftrightarrow \quad w^{-} \leqslant \tilde{V}(\tau)+\tilde{p}(\tau)
$$

Then

$$
\begin{gathered}
\tilde{V}(\check{\tau}) \leqslant v^{-} \leqslant v^{+} \leqslant \tilde{V}(\tau)\left(1-\frac{\Delta t}{\delta}\right)+\frac{\Delta t}{\delta} \tilde{V}(\tau) \leqslant \tilde{V}(\hat{\tau}) \\
\tilde{V}(\hat{\tau})+\tilde{p}(\hat{\tau}) \leqslant w^{-} \leqslant w^{+} \leqslant \tilde{V}(\tau)+\tilde{p}(\tau) \leqslant \tilde{V}(\check{\tau})+\tilde{p}(\check{\tau}),
\end{gathered}
$$

thus $u^{+} \in \mathbf{E}$.

The uniform $T V$ bound is provided by the following lemma.

Lemma 2.2. Assume that the strict sub-characteristic condition (1.11) is satisfied. For $\Delta t \leqslant \delta$, the total variation of the Riemann invariants of the constructed approximation $U^{\nu}$ is nonincreasing in time:

$$
T V\left(w^{\nu}(t, \cdot)\right)+T V\left(v\left(U^{\nu}(t, \cdot)\right)\right) \leqslant T V\left(w_{0}^{\nu}\right)+T V\left(v\left(U_{0}^{\nu}\right)\right), \quad \text { for a.e. } t>0 .
$$

Proof. When solving the homogeneous system in step 2, the total variation of both Riemann invariants is non increasing in time since we are dealing with a Temple system [7]. We thus focus on the evolution of the total variation at step 3 . We recall that

$$
\begin{aligned}
\tau\left(t_{n}^{+}, x\right) & =\tau\left(t_{n}^{-}, x\right), \\
w\left(t_{n}^{+}, x\right) & =w\left(t_{n}^{-}, x\right)+\frac{\Delta t}{\delta}(\tilde{V}(\tau)-w+\tilde{p}(\tau))\left(t_{n}^{-}, x\right) .
\end{aligned}
$$

Therefore

$$
\begin{aligned}
\left|w_{r}^{+}-w_{l}^{+}\right| & =\left|\left(w_{r}^{-}-w_{l}^{-}\right)+\frac{\Delta t}{\delta}\left[\left(\tilde{V}\left(\tau_{r}\right)-\tilde{V}\left(\tau_{l}\right)\right)+\left(\tilde{p}\left(\tau_{r}\right)-\tilde{p}\left(\tau_{l}\right)\right)-\left(w_{r}^{-}-w_{l}^{-}\right)\right]\right| \\
\left|v_{r}^{+}-v_{l}^{+}\right| & =\left|\left(w_{r}^{+}-w_{l}^{+}\right)-\left(\tilde{p}\left(\tau_{r}\right)-\tilde{p}\left(\tau_{l}\right)\right)\right|,
\end{aligned}
$$




$$
=\left|\left(w_{r}^{-}-w_{l}^{-}\right)+\frac{\Delta t}{\delta}\left[\left(\tilde{V}\left(\tau_{r}\right)-\tilde{V}\left(\tau_{l}\right)\right)+\left(\tilde{p}\left(\tau_{r}\right)-\tilde{p}\left(\tau_{l}\right)\right)-\left(w_{r}^{-}-w_{l}^{-}\right)\right]-\left(\tilde{p}\left(\tau_{r}\right)-\tilde{p}\left(\tau_{l}\right)\right)\right| .
$$

We distinguish three cases, depending on the wave arriving at $t=t_{n}^{-}$.

1-rarefaction wave. We denote by $U_{l}$ and $U_{r}$ respectively the left and right states of the wave hitting the line $t=t_{n}$ at $t=t_{n}^{-}$. Let us consider first the case where $U_{l}$ and $U_{r}$ are connected through a rarefaction wave. This implies $w_{l}^{-}=w_{r}^{-}$and $\tau_{l}<\tau_{r}$ (and $v_{l}^{-}<v_{r}^{-}$). In addition, $\tilde{V}$ is an increasing function of $\tau$, ie $\tilde{V}\left(\tau_{l}\right)<\tilde{V}\left(\tau_{r}\right)$. The same way, $\tilde{p}$ is a decreasing function of $\tau$. Thus $\tilde{p}\left(\tau_{l}\right)>\tilde{p}\left(\tau_{r}\right)$. We have:

$$
\begin{aligned}
& \left|w_{r}^{+}-w_{l}^{+}\right|=\frac{\Delta t}{\delta}|\underbrace{\left(\tilde{V}\left(\tau_{r}\right)-\tilde{V}\left(\tau_{l}\right)\right)}_{\geqslant 0}+\underbrace{\left(\tilde{p}\left(\tau_{r}\right)-\tilde{p}\left(\tau_{l}\right)\right)}_{\leqslant 0}|, \\
& \left|v_{r}^{+}-v_{l}^{+}\right|=|\frac{\Delta t}{\delta} \underbrace{\left(\tilde{V}\left(\tau_{r}\right)-\tilde{V}\left(\tau_{l}\right)\right)}_{\geqslant 0}+\left(1-\frac{\Delta t}{\delta}\right) \underbrace{\left(\tilde{p}\left(\tau_{l}\right)-\tilde{p}\left(\tau_{r}\right)\right)}_{\geqslant 0}| .
\end{aligned}
$$

In addition:

$$
\left|w_{r}^{-}-w_{l}^{-}\right|+\left|v_{r}^{-}-v_{l}^{-}\right|=\left|v_{r}^{-}-v_{l}^{-}\right|=\tilde{p}\left(\tau_{l}\right)-\tilde{p}\left(\tau_{r}\right) .
$$

By applying the sub-characteristic condition (1.11), we have:

$$
\begin{aligned}
& \tilde{p}\left(\tau_{r}\right)-\tilde{p}\left(\tau_{l}\right)=\int_{\tau_{l}}^{\tau_{r}} \underbrace{\tilde{p}^{\prime}(\tau)}_{\leqslant 0} d \tau, \\
& \tilde{p}\left(\tau_{l}\right)-\tilde{p}\left(\tau_{r}\right)=\int_{\tau_{l}}^{\tau_{r}}\left|\tilde{p}^{\prime}(\tau)\right| d \tau \geqslant \int_{\tau_{l}}^{\tau_{r}}|\underbrace{\tilde{V}^{\prime}(\tau)}_{\geqslant 0}| d \tau=\tilde{V}\left(\tau_{r}\right)-\tilde{V}\left(\tau_{l}\right) .
\end{aligned}
$$

Then:

$$
\begin{aligned}
\left|w_{r}^{+}-w_{l}^{+}\right| & =\frac{\Delta t}{\delta}\left(\left(\tilde{p}\left(\tau_{l}\right)-\tilde{p}\left(\tau_{r}\right)\right)-\left(\tilde{V}\left(\tau_{r}\right)-\tilde{V}\left(\tau_{l}\right)\right)\right) \\
& =\frac{\Delta t}{\delta}\left(\left(v_{r}^{-}-v_{l}^{-}\right)-\left(\tilde{V}\left(\tau_{r}\right)-\tilde{V}\left(\tau_{l}\right)\right)\right) .
\end{aligned}
$$

Since $\Delta t \leqslant \delta$, we get

$$
\left|v_{r}^{+}-v_{l}^{+}\right|=\frac{\Delta t}{\delta}\left(\tilde{V}\left(\tau_{r}\right)-\tilde{V}\left(\tau_{l}\right)\right)+\left(1-\frac{\Delta t}{\delta}\right)\left|v_{r}^{-}-v_{l}^{-}\right| .
$$

Finally,

$$
\left|w_{r}^{+}-w_{l}^{+}\right|+\left|v_{r}^{+}-v_{l}^{+}\right|=\left|w_{r}^{-}-w_{l}^{-}\right|+\left|v_{r}^{-}-v_{l}^{-}\right|,
$$

thus the total variation of the Riemann invariants is conserved through the splitting step.

1-shock wave. We now assume that $U_{l}$ and $U_{r}$ are connected by a shock. This means that $w_{l}^{-}=w_{r}^{-}$and $\tau_{l}>\tau_{r}$. We also have, by monotonicity of $\tilde{V}$ and $\tilde{p}, \tilde{V}\left(\tau_{l}\right)>\tilde{V}\left(\tau_{r}\right)$ and $\tilde{p}\left(\tau_{l}\right)<\tilde{p}\left(\tau_{r}\right)$. We can apply the same computations as before:

$$
\left|w_{r}^{+}-w_{l}^{+}\right|=\frac{\Delta t}{\delta}|\underbrace{\left(\tilde{V}\left(\tau_{r}\right)-\tilde{V}\left(\tau_{l}\right)\right)}_{\leqslant 0}+\underbrace{\left(\tilde{p}\left(\tau_{r}\right)-\tilde{p}\left(\tau_{l}\right)\right)}_{\geqslant 0}|
$$




$$
\left|v_{r}^{+}-v_{l}^{+}\right|=|\frac{\Delta t}{\delta} \underbrace{\left(\tilde{V}\left(\tau_{r}\right)-\tilde{V}\left(\tau_{l}\right)\right)}_{\leqslant 0}+\left(1-\frac{\Delta t}{\delta}\right) \underbrace{\left(\tilde{p}\left(\tau_{l}\right)-\tilde{p}\left(\tau_{r}\right)\right)}_{\leqslant 0}| .
$$

In addition,

$$
\left|w_{r}^{-}-w_{l}^{-}\right|+\left|v_{r}^{-}-v_{l}^{-}\right|=\left|v_{r}^{-}-v_{l}^{-}\right|=\tilde{p}\left(\tau_{r}\right)-\tilde{p}\left(\tau_{l}\right) .
$$

The sub-characteristic condition still yields that :

$$
\tilde{p}\left(\tau_{r}\right)-\tilde{p}\left(\tau_{l}\right) \geqslant \tilde{V}\left(\tau_{l}\right)-\tilde{V}\left(\tau_{r}\right) .
$$

Thus we have

$$
\begin{aligned}
\left|w_{r}^{+}-w_{l}^{+}\right| & =\frac{\Delta t}{\delta}\left(\left(\tilde{V}\left(\tau_{r}\right)-\tilde{V}\left(\tau_{l}\right)\right)+\left(\tilde{p}\left(\tau_{r}\right)-\tilde{p}\left(\tau_{l}\right)\right)\right) \\
\left|v_{r}^{+}-v_{l}^{+}\right| & =-\left(\frac{\Delta t}{\delta}\left(\tilde{V}\left(\tau_{r}\right)-\tilde{V}\left(\tau_{l}\right)\right)+\left(1-\frac{\Delta t}{\delta}\right)\left(\tilde{p}\left(\tau_{l}\right)-\tilde{p}\left(\tau_{r}\right)\right)\right) .
\end{aligned}
$$

Finally,

$$
\left|w_{r}^{+}-w_{l}^{+}\right|+\left|v_{r}^{+}-v_{l}^{+}\right|=\left|w_{r}^{-}-w_{l}^{-}\right|+\left|v_{r}^{-}-v_{l}^{-}\right| .
$$

2-contact discontinuity. We now consider the case where $U_{l}$ and $U_{r}$ are connected through a 2-contact discontinuity, which means $v_{l}^{-}=v_{r}^{-}$. We compute

$$
\begin{aligned}
\left|w_{r}^{+}-w_{l}^{+}\right| & =\left|\left(\tilde{p}\left(\tau_{r}\right)-\tilde{p}\left(\tau_{l}\right)\right)+\frac{\Delta t}{\delta}\left(\tilde{V}\left(\tau_{r}\right)-\tilde{V}\left(\tau_{l}\right)\right)\right|, \\
\left|v_{r}^{+}-v_{l}^{+}\right| & =\left|\left(w_{r}^{-}-w_{l}^{-}\right)+\frac{\Delta t}{\delta}\left(\tilde{V}\left(\tau_{r}\right)-\tilde{V}\left(\tau_{l}\right)\right)-\left(\tilde{p}\left(\tau_{r}\right)-\tilde{p}\left(\tau_{l}\right)\right)\right|=\frac{\Delta t}{\delta}\left|\tilde{V}\left(\tau_{r}\right)-\tilde{V}\left(\tau_{l}\right)\right| .
\end{aligned}
$$

In addition

$$
\left|w_{r}^{-}-w_{l}^{-}\right|+\left|v_{r}^{-}-v_{l}^{-}\right|=\left|w_{r}^{-}-w_{l}^{-}\right|=\left|\tilde{p}\left(\tau_{r}\right)-\tilde{p}\left(\tau_{l}\right)\right| .
$$

We note that

$$
\left(\tilde{p}\left(\tau_{r}\right)-\tilde{p}\left(\tau_{l}\right)\right) \cdot\left(\tilde{V}\left(\tau_{r}\right)-\tilde{V}\left(\tau_{l}\right)\right) \leqslant 0 .
$$

Since $\Delta t \leqslant \delta$, we have:

$$
\begin{aligned}
\left|w_{r}^{+}-w_{l}^{+}\right| & =\operatorname{sgn}\left(\tilde{p}\left(\tau_{r}\right)-\tilde{p}\left(\tau_{l}\right)\right)\left(\left(\tilde{p}\left(\tau_{r}\right)-\tilde{p}\left(\tau_{l}\right)\right)+\frac{\Delta t}{\delta}\left(\tilde{V}\left(\tau_{r}\right)-\tilde{V}\left(\tau_{l}\right)\right)\right), \\
\left|v_{r}^{+}-v_{l}^{+}\right| & =-\operatorname{sgn}\left(\tilde{p}\left(\tau_{r}\right)-\tilde{p}\left(\tau_{l}\right)\right) \frac{\Delta t}{\delta}\left(\tilde{V}\left(\tau_{r}\right)-\tilde{V}\left(\tau_{l}\right)\right),
\end{aligned}
$$

and thus

$$
\left|w_{r}^{+}-w_{l}^{+}\right|+\left|v_{r}^{+}-v_{l}^{+}\right|=\left|w_{r}^{-}-w_{l}^{-}\right|+\left|v_{r}^{-}-v_{l}^{-}\right| .
$$


Lemma 2.3. Let $\nu \in \mathbb{N}$ and $U_{0}^{\nu} \in \mathcal{D}(M)$. Then there exist a constant $C_{M}$ independent of $\delta$, and a constant $L_{\delta}$ such that, $\forall a<b, \forall 0 \leqslant s<t$ :

$$
\begin{aligned}
& \int_{a}^{b}\left|\tau^{\nu}(t, X)-\tau^{\nu}(s, X)\right| d X \leqslant C_{M}(t-s) \\
& \int_{a}^{b}\left|w^{\nu}(t, X)-w^{\nu}(s, X)\right| d X \leqslant\left(C_{M}+L_{\delta}\right)(t-s+\Delta t) .
\end{aligned}
$$

Proof. Lemmas 2.1 and 2.2 imply that $U^{\nu}(t, \cdot) \in \mathcal{D}(M)$ for all $t>0$. Let $s, t \in \mathbb{R}$ such that $0 \leqslant s<t$. If there are no time-steps between $s$ and $t,(2.6)$ and (2.7) are true for any $L_{\delta} \geqslant 0$, as a direct application of Temple-class system properties, see [39, Theorem 13.3.1] and [7, Theorem $1]$.

Suppose now that there are $N+1$ time-steps between $s$ and $t$ :

$$
s \leqslant k \Delta t \leqslant(k+1) \Delta t \leqslant \cdots \leqslant(k+N) \Delta t \leqslant t \quad \text { for } \geqslant 1,
$$

so that $N \Delta t \leqslant t-s$.

Let $a<b$ given and $X \in] a, b[$. We can then write:

$$
\begin{aligned}
\left|\tau^{\nu}(t, X)-\tau^{\nu}(s, X)\right|=\mid \tau^{\nu}(t, X)-\tau^{\nu}((k+N) \Delta t, X) & +\sum_{i=k}^{k+N-1}\left(\tau^{\nu}((i+1) \Delta t, X)-\tau^{\nu}(i \Delta t, X)\right) \\
& +\tau^{\nu}(k \Delta t, X)-\tau^{\nu}(s, X) \mid \\
\leqslant\left|\tau^{\nu}(t, X)-\tau^{\nu}((k+N) \Delta t, X)\right| & +\sum_{i=k}^{k+N-1}\left|\tau^{\nu}((i+1) \Delta t, X)-\tau^{\nu}(i \Delta t, X)\right| \\
& +\left|\tau^{\nu}(k \Delta t, X)-\tau^{\nu}(s, X)\right| .
\end{aligned}
$$

Since $\tau^{\nu}$ does not change through the splitting process, we can apply the previous property between two consecutive time-steps to obtain (2.6):

$$
\begin{aligned}
\int_{a}^{b}\left|\tau^{\nu}(t, X)-\tau^{\nu}(s, X)\right| d X & \leqslant C_{M}\left[(t-(k+N) \Delta t)+\sum_{i=k}^{k+N-1} \Delta t+(k \Delta t-s)\right] \\
& \leqslant C_{M}(t-s) .
\end{aligned}
$$

For the second inequality, we have to consider an additional term, since $w^{\nu}$ is modified at each splitting-step. We can then write:

$$
\begin{aligned}
\sum_{i=k}^{k+N} \int_{a}^{b}\left|w^{\nu}(i \Delta t+, X)-w^{\nu}(i \Delta t-, X)\right| d X & =\sum_{i=k}^{k+N} \frac{\Delta t}{\delta} \int_{a}^{b}\left|\tilde{V}\left(\tau^{\nu}(i \Delta t-, X)\right)-v(i \Delta t-, X)\right| d X \\
& \leqslant \frac{\Delta t}{\delta}(N+1)(b-a) \sup _{U^{\nu} \in \mathcal{D}(M)}\left|\tilde{V}\left(\tau^{\nu}\right)-\left(w^{\nu}-\tilde{p}\left(\tau^{\nu}\right)\right)\right| \\
& \leqslant L_{\delta}(t-s+\Delta t),
\end{aligned}
$$

where

$$
L_{\delta}=\frac{(b-a)}{\delta} \sup _{U^{\nu} \in \mathcal{D}(M)}\left|\tilde{V}\left(\tau^{\nu}\right)-\left(w^{\nu}-\tilde{p}\left(\tau^{\nu}\right)\right)\right| .
$$

Summing this term with the estimates that remain valid between two consecutive time-steps, we obtain (2.7). 


\section{Convergence of the WFT approximate solutions to a solution of the relaxed ARZ system}

The following theorem ensures the existence of weak entropy solutions of the Cauchy problem (1.9), 1.10 .

Theorem 3.1. Let $U_{0}=\left(\tau_{0}, w_{0}\right) \in \mathcal{D}(M)$ for some $M>0$ and, for any $\delta>0$ fixed, denote by $U^{\delta}=\left(\tau^{\delta}, w^{\delta}\right)$ the limit of a subsequence $U^{\nu}=\left(\tau^{\nu}, w^{\nu}\right)$ of WFT approximate solutions as $\nu \rightarrow \infty$. Then $U^{\delta}$ is a weak entropic solution of 1.9 , (1.10).

Proof. The proof follows the guidelines of [19]. The existence of the limit $U^{\delta}$ and the convergence in $L_{\text {loc }}^{1}\left(\left[0,+\infty[\times \mathbb{R})\right.\right.$ is guaranteed by [8, Theorem 2.4]. Moreover, each component of $U^{\delta}$ satisfies (2.6) and (2.7). For simplicity of notations, we will drop the $\delta$ index in the proof and name $U$ the limit of a subsequence of WFT approximate solutions.

System $(1.9)$ can be rewritten as:

$$
U_{t}+[F(U)]_{X}=G(U)
$$

with

$$
U=\left(\begin{array}{c}
\tau \\
w
\end{array}\right), \quad F(U)=\left(\begin{array}{c}
-(w-\tilde{p}(\tau)) \\
0
\end{array}\right), \quad G(U)=\left(\begin{array}{c}
0 \\
\frac{\tilde{V}(\tau)-(w-\tilde{p}(\tau))}{\delta}
\end{array}\right)
$$

Let $T>0$ a given finite time-horizon. Let $\phi \in \mathcal{C}_{c}^{1}([0, T[\times \mathbb{R})$. To be a weak solution of $[1.9], U$ must satisfy:

$$
\begin{array}{r}
\int_{-\infty}^{+\infty} \phi(0, X) U_{0}(X) d X+\int_{0}^{T} \int_{-\infty}^{+\infty}\left[\phi_{t}(t, X) U(t, X)+\phi_{X}(t, X) F(U(t, X))\right] d X d t \\
+\int_{0}^{T} \int_{-\infty}^{+\infty} \phi(t, X) G(U(t, X)) d X d t=0
\end{array}
$$

We define $N^{\nu} \in \mathbb{N}$ such that $T=N^{\nu} \Delta t^{\nu}+\beta^{\nu}, \beta^{\nu} \in\left[0, \Delta t^{\nu}\right.$. We will proceed component by component. Let us begin with $\tau$.

For each $k \in\left\{0, . ., N_{\nu}-1\right\}$, since $\tau^{\nu}$ satisfies the first equation of 1.9 we have:

$$
\begin{aligned}
\int_{k \Delta t^{\nu}}^{(k+1) \Delta t^{\nu}} & \int_{-\infty}^{+\infty}\left[\phi_{t} \tau^{\nu}-\phi_{X}\left(w^{\nu}-\tilde{p}\left(\tau^{\nu}\right)\right)\right] d X d t=\int_{-\infty}^{+\infty} \phi\left((k+1) \Delta t^{\nu}, X\right) \tau^{\nu}\left((k+1) \Delta t^{\nu}-, X\right) d X \\
& -\int_{-\infty}^{+\infty} \phi\left(k \Delta t^{\nu}, X\right) \tau^{\nu}\left(k \Delta t^{\nu}+, X\right) d X
\end{aligned}
$$

Then

$$
\begin{aligned}
& \int_{0}^{T} \int_{-\infty}^{+\infty}\left[\phi_{t} \tau^{\nu}-\phi_{X}\left(w^{\nu}-\tilde{p}\left(\tau^{\nu}\right)\right)\right] d X d t= \\
& \sum_{k=0}^{N^{\nu}-1} \int_{k \Delta t^{\nu}}^{(k+1) \Delta t^{\nu}} \int_{-\infty}^{+\infty}\left[\phi_{t} \tau^{\nu}-\phi_{X}\left(w^{\nu}-\tilde{p}\left(\tau^{\nu}\right)\right)\right] d X d t+\int_{N^{\nu} \Delta t^{\nu}}^{T} \int_{-\infty}^{+\infty}\left[\phi_{t} \tau^{\nu}-\phi_{X}\left(w^{\nu}-\tilde{p}\left(\tau^{\nu}\right)\right)\right] d X d t \\
= & \int_{-\infty}^{+\infty} \phi\left(N^{\nu} \Delta t^{\nu}, X\right) \tau^{\nu}\left(N^{\nu} \Delta t^{\nu}-, X\right) d X-\int_{-\infty}^{+\infty} \phi(0, X) \tau^{\nu}(0+, X) d X \\
& +\int_{N^{\nu} \Delta t^{\nu}}^{T} \int_{-\infty}^{+\infty}\left[\phi_{t} \tau^{\nu}-\phi_{X}\left(w^{\nu}-\tilde{p}\left(\tau^{\nu}\right)\right)\right] d X d t .
\end{aligned}
$$


Thus, by reordering,

$$
\begin{aligned}
& \int_{-\infty}^{+\infty} \phi(0, X) \tau_{0}(X) d X+\int_{0}^{T} \int_{-\infty}^{+\infty}\left[\phi_{t} \tau^{\nu}-\phi_{X}\left(w^{\nu}-\tilde{p}\left(\tau^{\nu}\right)\right)\right] d X d t= \\
& \int_{-\infty}^{+\infty} \phi\left(N^{\nu} \Delta t^{\nu}, X\right) \tau^{\nu}\left(N^{\nu} \Delta t^{\nu}-, X\right) d X+\int_{N^{\nu} \Delta t^{\nu}}^{T} \int_{-\infty}^{+\infty}\left[\phi_{t} \tau^{\nu}-\phi_{X}\left(w^{\nu}-\tilde{p}\left(\tau^{\nu}\right)\right)\right](t, X) d X d t .
\end{aligned}
$$

Since $T-N^{\nu} \Delta t^{\nu}<\Delta t^{\nu}$, and since $\phi$ has compact support, there exist $\eta$ such that:

$$
\phi(t, X)=0, \quad \forall \nu>\eta, \forall X \in \mathbb{R}, \forall t \geqslant N^{\nu} \Delta t^{\nu} .
$$

Thus the right hand side converges to 0 when $\nu \rightarrow \infty$ and then $\tau$ satisfies (3.1).

Let us now consider $w$. The approximate function $w^{\nu}$ satisfies:

$$
\begin{aligned}
\int_{k \Delta t^{\nu}}^{(k+1) \Delta t^{\nu}} \int_{-\infty}^{+\infty} \phi_{t} w^{\nu} d X d t= & \int_{-\infty}^{+\infty} \phi\left((k+1) \Delta t^{\nu}, X\right) w^{\nu}\left((k+1) \Delta t^{\nu}-, X\right) d X \\
& -\int_{-\infty}^{+\infty} \phi\left(k \Delta t^{\nu}, X\right) w^{\nu}\left(k \Delta t^{\nu}+, X\right) d X \\
= & \int_{-\infty}^{+\infty} \phi\left((k+1) \Delta t^{\nu}, X\right) w^{\nu}\left((k+1) \Delta t^{\nu}-, X\right) d X \\
& -\int_{-\infty}^{+\infty} \phi\left(k \Delta t^{\nu}, X\right)\left[w^{\nu}\left(k \Delta t^{\nu}-, X\right)+\Delta t^{\nu} \frac{\tilde{V}\left(\tau^{\nu}\right)-v^{\nu}}{\delta}\left(k \Delta t^{\nu}-, X\right)\right] d X .
\end{aligned}
$$

Then we get

$$
\begin{aligned}
\int_{0}^{T} \int_{-\infty}^{+\infty} \phi_{t} w^{\nu} d X d t= & \sum_{k=0}^{N^{\nu}-1} \int_{-\infty}^{+\infty}\left[\phi\left((k+1) \Delta t^{\nu}, X\right) w^{\nu}\left((k+1) \Delta t^{\nu}-, X\right)-\phi\left(k \Delta t^{\nu}, X\right) w^{\nu}\left(k \Delta t^{\nu}-, X\right)\right] d X \\
& -\sum_{k=0}^{N^{\nu}-1} \int_{-\infty}^{+\infty} \phi\left(k \Delta t^{\nu}, X\right) \Delta t \frac{\tilde{V}\left(\tau^{\nu}\right)-v^{\nu}}{\delta}\left(k \Delta t^{\nu}-, X\right) d X \\
& +\int_{N^{\nu} \Delta t^{\nu}}^{T} \int_{-\infty}^{+\infty} \phi_{t} w^{\nu} d X d t \\
= & \int_{-\infty}^{+\infty} \phi\left(N^{\nu} \Delta t^{\nu}, X\right) w^{\nu}\left(N^{\nu} \Delta t^{\nu}-, X\right) d X-\int_{-\infty}^{+\infty} \phi(0, X) w_{0}(X) d X \\
& -\sum_{k=0}^{N^{\nu}-1} \int_{-\infty}^{+\infty} \phi\left(k \Delta t^{\nu}, X\right) \Delta t \frac{\tilde{V}\left(\tau^{\nu}\right)-v^{\nu}}{\delta}\left(k \Delta t^{\nu}-, X\right) d X \\
& +\int_{N^{\nu} \Delta t^{\nu}}^{T} \int_{-\infty}^{+\infty} \phi_{t} w^{\nu} d X d t
\end{aligned}
$$

By the same argument as before, picking $\Delta t^{\nu}$ small enough, we have:

$$
\int_{-\infty}^{+\infty} \phi\left(N^{\nu} \Delta t^{\nu}, X\right) w^{\nu}\left(N^{\nu} \Delta t^{\nu}-, X\right) d X+\int_{N^{\nu} \Delta t^{\nu}}^{T} \int_{-\infty}^{+\infty} \phi_{t} w^{\nu} d X d t=0 .
$$

Let us define the sequence of functions $\psi^{\nu}$ such that

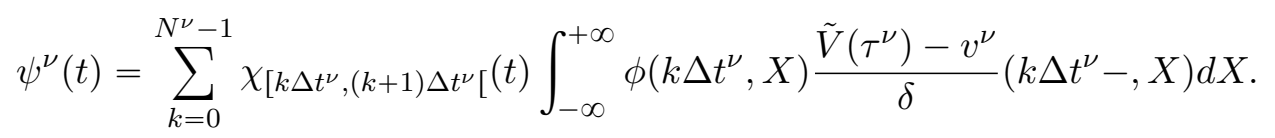


Setting for any $(\tau, v) \in \mathbb{R}_{+}^{2}$

$$
g_{\nu}(t, X, \tau, v)=\sum_{k=0}^{N^{\nu}-1} \chi_{\left[k \Delta t^{\nu},(k+1) \Delta t^{\nu}[\right.}(t) \frac{\tilde{V}(\tau)-v}{\delta}\left(k \Delta t^{\nu}-, X\right),
$$

we observe that $g_{\nu}$ converges uniformly to $\frac{\tilde{V}(\tau)-v}{\delta}$ on compact subsets of $[0, T] \times \mathbb{R} \times \mathbb{R}_{+}^{2}$. In addition, $\tau^{\nu} \rightarrow \tau$ and $v^{\nu} \rightarrow v$ in $\mathbb{L}_{l o c}^{1}$. Thus, for almost every $t \in[0, T]$ :

$$
\psi^{\nu}(t) \rightarrow \int_{-\infty}^{+\infty} \phi(t, X) \frac{\tilde{V}(\tau)-v}{\delta}(t, X) d X=\psi(t) .
$$

We notice that

$$
\begin{aligned}
& \int_{0}^{T} \psi^{\nu}(t) d t=\int_{0}^{T} \sum_{k=0}^{N^{\nu}-1} \chi_{\left[k \Delta t^{\nu},(k+1) \Delta t^{\nu}[\right.}(t) \int_{-\infty}^{+\infty} \phi\left(k \Delta t^{\nu}, X\right) \frac{\tilde{V}\left(\tau^{\nu}\right)-v^{\nu}}{\delta}\left(k \Delta t^{\nu}-, X\right) d X d t \\
& =\sum_{k=0}^{N^{\nu}-1} \int_{0}^{T} \chi_{\left[k \Delta t^{\nu},(k+1) \Delta t^{\nu}[\right.}(t) d t \int_{-\infty}^{+\infty} \phi\left(k \Delta t^{\nu}, X\right) \frac{\tilde{V}\left(\tau^{\nu}\right)-v^{\nu}}{\delta}\left(k \Delta t^{\nu}-, X\right) d X \\
& =\sum_{k=0}^{N^{\nu}-1} \Delta t^{\nu} \int_{-\infty}^{+\infty} \phi\left(k \Delta t^{\nu}, X\right) \frac{\tilde{V}\left(\tau^{\nu}\right)-v^{\nu}}{\delta}\left(k \Delta t^{\nu}-, X\right) d X .
\end{aligned}
$$

By the Lebesgue dominated convergence theorem,

$$
\lim _{\Delta t^{\nu} \rightarrow 0} \int_{0}^{T} \psi^{\nu}(t) d t=\int_{0}^{T} \psi(t) d t
$$

Passing to the limit in $(3.3)$ we recover

$$
\begin{array}{r}
\int_{-\infty}^{+\infty} \phi(0, X) w_{0}(X) d X+\int_{0}^{T} \int_{-\infty}^{+\infty} \phi_{t}(t, X) w(t, X) d X d t \\
+\int_{0}^{T} \int_{-\infty}^{+\infty} \phi(t, X) \frac{\tilde{V}(\tau)-v}{\delta} d X d t=0 .
\end{array}
$$

Therefore the limit $U$ of our sequence of approximate solutions is a weak solution of 1.9 . Let us prove that $U$ is an entropic solution as well. We fix a smooth convex entropy $\eta$ with associated flux $q$. By definition, it satisfies:

$$
\begin{aligned}
& \nabla \eta^{T}(z) D F(z)=\nabla^{T} q(z), \quad \text { for } z \in \mathbb{R}_{+}^{2} . \\
& \nabla \eta^{T}(z) G(z) \leqslant 0,
\end{aligned}
$$

Existence of such an entropy, entropy-flux pair is guaranteed by [14, Theorem 3.2].

Let $\phi \in \mathcal{C}_{c}^{1}([0, T[, \mathbb{R}), \phi \geqslant 0$. We need to show that

$$
\begin{aligned}
& \int_{0}^{T} \int_{-\infty}^{+\infty}\left[\eta(U(t, X)) \phi_{t}(t, X)+q(U(t, X)) \phi_{x}(t, X)\right. \\
& \left.+\nabla \eta^{T}(U(t, X)) G(t, X, U(t, X)) \phi(t, X)\right] d X d t+\int_{-\infty}^{+\infty} \phi(0, X) \eta\left(U_{0}(X)\right) d X \geqslant 0 .
\end{aligned}
$$

As we did in $(3.3)$, we can write:

$$
\int_{0}^{T} \int_{-\infty}^{+\infty}\left[\eta\left(U^{\nu}(t, X)\right) \phi_{t}(t, X)+q\left(U^{\nu}(t, X)\right) \phi_{x}(t, X)\right] d X d t
$$




$$
\begin{aligned}
\geqslant & \sum_{k=0}^{N^{\nu}-1} \int_{-\infty}^{+\infty}\left[\eta\left(U^{\nu}\left((k+1) \Delta t^{\nu}-, X\right)\right) \phi\left((k+1) \Delta t^{\nu}-, X\right)-\eta\left(U^{\nu}\left(k \Delta t^{\nu}+, X\right)\right) \phi\left(k \Delta t^{\nu}+, X\right)\right] d X \\
& +\int_{N^{\nu} \Delta t^{\nu}}^{T} \int_{-\infty}^{+\infty}\left[\eta\left(U^{\nu}(t, X)\right) \phi_{t}(t, X)+q\left(U^{\nu}(t, X)\right) \phi_{x}(t, X)\right] d X d t+O\left(\epsilon_{\nu}\right) .
\end{aligned}
$$

By the same compactness argument than before, we state that the last integral is identically zero for $\Delta t^{\nu}$ small enough. 
The remaining terms give

$$
\begin{aligned}
& \int_{0}^{T} \int_{-\infty}^{+\infty}\left[\eta\left(U^{\nu}(t, X)\right) \phi_{t}(t, X)+q\left(U^{\nu}(t, X)\right) \phi_{x}(t, X)\right] d X d t \\
\geqslant & \sum_{k=1}^{N^{\nu}} \int_{-\infty}^{+\infty}\left[\eta\left(U^{\nu}\left(k \Delta t^{\nu}-, X\right)\right) \phi\left(k \Delta t^{\nu}-, X\right)-\eta\left(U^{\nu}\left(k \Delta t^{\nu}+, X\right)\right) \phi\left(k \Delta t^{\nu}+, X\right)\right] d X \\
& +\int_{-\infty}^{+\infty} \eta\left(U^{\nu}\left(N^{\nu} \Delta t^{\nu}+, X\right)\right) \underbrace{\phi\left(N^{\nu} \Delta t^{\nu}+, X\right)}_{=0} d X-\int_{-\infty}^{+\infty} \phi(0, X) \eta\left(U_{0}(X)\right) d X+\mathcal{O}\left(\epsilon_{\nu}\right) \\
\geqslant & -\Delta t^{\nu} \sum_{k=1}^{N^{\nu}} \int_{-\infty}^{+\infty} \partial_{w} \eta\left(U^{\nu}\left(k \Delta t^{\nu}+, X\right)\right) \frac{\tilde{V}\left(\tau^{\nu}\right)-v^{\nu}}{\delta}\left(k \Delta t^{\nu}-, X\right) \phi\left(k \Delta t^{\nu}, X\right) d X \\
& -\int_{-\infty}^{+\infty} \phi(0, X) \eta\left(U_{0}(X)\right) d X+\mathcal{O}\left(\epsilon_{\nu}\right) .
\end{aligned}
$$

With the same reasoning as before we pass to the limit using the Dominated Convergence Theorem, obtaining the desired inequality (3.5).

\section{Estimates of positive waves}

Oleŭnik type entropy estimates [34, 35] are known for scalar equations [26], genuinely nonlinear systems [9, 10, 12, in particular of Temple class [3, 11], and also balance laws [16, 17, 22. In this section, we provide a decay estimate for positive waves of the ARZ system with relaxation, accounting for the source term contribution.

Proposition 4.1. Assume that $\exists c_{0}>0$ such that $\forall u, u^{\prime} \in \mathbf{E}$ :

$$
\begin{aligned}
& \left|\lambda_{1}(u)-\lambda_{1}\left(u^{\prime}\right)\right|=\left|\tilde{p}^{\prime}(\tau)-\tilde{p}^{\prime}\left(\tau^{\prime}\right)\right| \leqslant c_{0}, \\
& \left|\lambda_{1}(u)-\lambda_{2}\left(u^{\prime}\right)\right|=\left|\tilde{p}^{\prime}(\tau)\right| \geqslant 2 c_{0} .
\end{aligned}
$$

Then, there exists a constant $C>0$ such that, for any interval $] a, b[$, for any time horizon $T>0$, and every initial condition $(1.10)$, the measure $\mu_{T}^{1+}(] a, b[)$ of positive 1-waves contained in the solution of (1.9) obtained as limit of the proposed Wave-Front-Tracking approximations satisfies

$$
\mu_{T}^{1+}(] a, b[) \leqslant C \frac{b-a}{T} e^{C \frac{T}{\delta}\left(T V\left(w_{0}\right)+T V\left(v_{0}\right)\right)}+C \frac{T}{\delta}\left(T V\left(w_{0}\right)+T V\left(v_{0}\right)\right) .
$$

Proof. Consider the sequence $U^{\nu}(t, x)=\left(\tau^{\nu}, w^{\nu}\right)(t, x)$ of piecewise constant approximate solution constructed by the WFT algorithm proposed in Section 2.1. In Riemann-invariant coordinates, we denote this approximate solution by $W^{\nu}=\left(v^{\nu}, w^{\nu}\right)$, and then $\tau^{\nu}=(\tilde{p})^{-1}\left(w^{\nu}-v^{\nu}\right)$. We recall that a backward 1-characteristic is an absolutely continuous curve $x=x(t)$ such that $\dot{x}(t) \in\left[\lambda_{1}\left(W^{\nu}(t, x(t)+)\right), \lambda_{1}\left(W^{\nu}(t, x(t)-)\right)\right]$, see[8, Section 10]. On each subinterval $\left[t_{n}, t_{n+1}\right]$, if $x(t)$ does not coincide with a wavefront $x_{\alpha}(t)$, then $\dot{x}(t)=\lambda_{1}\left(W^{\nu}(t, x(t))\right)$. Else we have $\dot{x}(t)=\dot{x}_{\alpha}(t)$. We call $y(t)$ a minimal backward 1-characteristic through a point $\bar{x}$ if

$$
y(t)=\min \{x(t), x \text { is a backward 1-characteristic, } x(T)=\bar{x}\} .
$$

Let us fix $T>0$ and an interval $] a, b]$, and call $t \mapsto a(t) ; t \mapsto b(t)$ the minimal backward 1characteristics passing through $a, b$ at time $T$. Let $I(t)=] a(t), b(t)]$. We call $x_{\alpha}(t)$ the position of wavefronts at time $t$. Each wavefront belongs to a family $k_{\alpha} \in 1,2$ with a size $\sigma_{\alpha}$. The first family is genuinely non-linear, thus we set $\sigma_{1}=\lambda_{1}\left(W^{\nu}\left(t, x_{\alpha}(t)+\right)\right)-\lambda_{1}\left(W^{\nu}\left(t, x_{\alpha}(t)-\right)\right)$. The second is linearly degenerated, then we take $\sigma_{2}=w^{\nu}\left(t, x_{\alpha}(t)+\right)-w^{\nu}\left(t, x_{\alpha}(t)-\right)$. 
Let $z(t):=b(t)-a(t) \geqslant 0$.

$$
\begin{aligned}
\dot{z}(t) & =\dot{b}(t)-\dot{a}(t) \\
& =\lambda_{1}\left(W^{\nu}(t, b(t)+)\right)-\lambda_{1}\left(W^{\nu}(t, a(t)+)\right) \\
& =\sum_{\substack{k_{\alpha}=1,2 \\
x_{\alpha}(t) \in I(t)}}\left[\lambda_{1}\left(W^{\nu}\left(t, x_{\alpha}(t)+\right)\right)-\lambda_{1}\left(W^{\nu}\left(t, x_{\alpha}(t)-\right)\right)\right] \\
& =\sum_{\substack{k_{\alpha}=1 \\
x_{\alpha}(t) \in I(t)}} \Delta \lambda_{1}\left(W^{\nu}\left(t, x_{\alpha}(t)\right)\right)+\sum_{\substack{k_{\alpha}=2 \\
x_{\alpha}(t) \in I(t)}} \Delta \lambda_{1}\left(W^{\nu}\left(t, x_{\alpha}(t)\right)\right) .
\end{aligned}
$$

Note that $\forall k_{\alpha}=2, x_{\alpha}(t) \in I(t)$,

$$
\begin{aligned}
\exists\left(v_{\alpha}, w_{\alpha}\right): \Delta \lambda_{1}\left(W^{\nu}\left(t, x_{\alpha}(t)\right)\right) & =\nabla_{v, w} \lambda_{1}\left(v_{\alpha}, w_{\alpha}\right) \cdot\left(W^{\nu}\left(t, x_{\alpha}(t)+\right)-W^{\nu}\left(t, x_{\alpha}(t)-\right)\right) \\
& =\frac{\tilde{p}^{\prime \prime}\left(\tilde{p}^{-1}\left(w_{\alpha}^{\nu}-v_{\alpha}^{\nu}\right)\right)}{\tilde{p}^{\prime}\left(\tilde{p}^{-1}\left(w_{\alpha}^{\nu}-v_{\alpha}^{\nu}\right)\right)}\left(w^{\nu}\left(t, x_{\alpha}(t)+\right)-w^{\nu}\left(t, x_{\alpha}(t)-\right)\right) .
\end{aligned}
$$

Since the domain $\mathbf{E}$ is compact, the mapping $W^{\nu} \rightarrow \lambda_{1}\left(W^{\nu}\right)$ is Lipschitz continuous with Lipschitz constant $C_{p}=\max _{\tau \in(\tilde{\tau}, \hat{\tau})}\left|\frac{\tilde{p}^{\prime \prime}(\tau)}{\tilde{p}^{\prime}(\tau)}\right|$, see 1.3$)$.

For almost every $t$ (outside splitting moments), from (4.3) we obtain

$$
\dot{z}(t) \geqslant M(t)-C_{p} K(t)
$$

with

$$
\begin{aligned}
M^{\nu}(t) & :=\sum_{\substack{k_{\alpha}=1 \\
x_{\alpha}(t) \in I(t)}} \sigma_{\alpha}=\sum_{\substack{k_{\alpha}=1 \\
x_{\alpha}(t) \in I(t)}} \Delta \lambda_{1}\left(W^{\nu}\left(t, x_{\alpha}(t)\right)\right), \\
K^{\nu}(t) & :=\sum_{\substack{k_{\alpha}=2 \\
x_{\alpha}(t) \in I(t)}}\left|\sigma_{\alpha}\right|=\sum_{\substack{k_{\alpha}=2 \\
x_{\alpha}(t) \in I(t)}}\left|w^{\nu}\left(t, x_{\alpha}(t)+\right)-w^{\nu}\left(t, x_{\alpha}(t)-\right)\right| .
\end{aligned}
$$

To estimate $K^{\nu}(t)$, let

$$
\Phi(t):=\sum_{\substack{k_{\alpha}=2 \\ x_{\alpha}(t) \in I(t)}} \phi\left(t, x_{\alpha}(t)\right)\left|\sigma_{\alpha}\right|,
$$

with

$$
\phi(t, x):= \begin{cases}1 & \text { if } x<a(t), \\ \frac{b(t)-x(t)}{z(t)} & \text { if } x \in[a(t), b(t)[, \\ 0 & \text { if } x \geqslant b(t) .\end{cases}
$$

Away from interaction points and splitting times the $\left|\sigma_{\alpha}\right|$ are constants and in $\left.] a(t), b(t)\right]$ we have by 4.1

$$
\begin{aligned}
\frac{d}{d t} \phi\left(t, x_{\alpha}(t)\right) & =\frac{\dot{b}(t)-\dot{x_{\alpha}}(t)}{z(t)}-\frac{\dot{z}(t)\left(b(t)-x_{\alpha}(t)\right)}{z(t)^{2}} \\
& \leqslant \frac{\dot{b}(t)-\dot{x_{\alpha}}(t)+|\dot{b}(t)-\dot{a}(t)|}{z(t)} \\
& \leqslant-\frac{c_{0}}{z(t)},
\end{aligned}
$$


therefore

$$
\dot{\Phi}(t) \leqslant-\frac{c_{0}}{z(t)} K^{\nu}(t)
$$

At interaction points (except splitting times), the quantity $\sum_{k_{\alpha}=2} \phi\left(t, x_{\alpha}(t)\right)\left|\sigma_{\alpha}\right|$ is constant, since wave strength does not change when measured in Riemann coordinates (and for a linearly degenerate field). Thus

$$
\dot{z}(t)-\frac{C_{p}}{c_{0}} z(t) \dot{\Phi}(t)-M^{\nu}(t) \geqslant 0 .
$$

We seek now for a uniform bound for $M^{\nu}(t)$ :

$$
M^{\nu}(t)=\sum_{\substack{k_{\alpha}=1 \\ x_{\alpha}(t) \in I(t)}}\left(\lambda_{1}\left(W^{\nu}\left(t, x_{\alpha}(t)+\right)\right)-\lambda_{1}\left(W^{\nu}\left(t, x_{\alpha}(t)-\right)\right)\right)
$$

Note that $\forall k_{\alpha}=1, x_{\alpha}(t) \in I(t)$,

$$
\begin{aligned}
\exists\left(v_{\alpha}, w_{\alpha}\right): \Delta \lambda_{1}\left(W^{\nu}\left(t, x_{\alpha}(t)\right)\right) & =\nabla_{v, w} \lambda_{1}\left(v_{\alpha}, w_{\alpha}\right) \cdot\left(W^{\nu}\left(t, x_{\alpha}(t)+\right)-W^{\nu}\left(t, x_{\alpha}(t)-\right)\right) \\
& =-\frac{\tilde{p}^{\prime \prime}\left(\tilde{p}^{-1}\left(w_{\alpha}^{\nu}-v_{\alpha}^{\nu}\right)\right)}{\tilde{p}^{\prime}\left(\tilde{p}^{-1}\left(w_{\alpha}^{\nu}-v_{\alpha}^{\nu}\right)\right)}\left(v^{\nu}\left(t, x_{\alpha}(t)+\right)-v^{\nu}\left(t, x_{\alpha}(t)-\right)\right)
\end{aligned}
$$

Now observe that

$$
\widetilde{M}^{\nu}(t):=\sum_{\substack{k_{\alpha}=1 \\ x_{\alpha}(t) \in I(t)}}\left(v^{\nu}\left(t, x_{\alpha}(t)+\right)-v^{\nu}\left(t, x_{\alpha}(t)-\right)\right)
$$

is constant except at splitting times, since we are in the case of a Temple class system, and is equivalent to $M^{\nu}$ again due to the Lipschitzianeity of the mapping $W \mapsto \lambda_{1}(W)$, see (1.3).

Let us estimate the changes occuring when the source term is integrated at a given splitting time $t=t_{n}=n \Delta t^{\nu}$, for a given $n$. Setting $g_{\delta}^{\nu}(t, x)=\frac{\left.\tilde{V}\left(\tilde{p}^{-1}\left(w^{\nu}-v^{\nu}\right)\right)\right)-v^{\nu}}{\delta}(x, t)$, we have:

$$
\begin{aligned}
\widetilde{M}^{\nu}\left(t_{n}+\right)= & \sum_{\begin{array}{c}
k_{\alpha}\left(t_{n}+\right)=1 \\
x_{\alpha}\left(t_{n}+\right) \in I\left(t_{n}+\right)
\end{array}}\left(v^{\nu}\left(t_{n}+, x_{\alpha}\left(t_{n}+\right)+\right)-v^{\nu}\left(t_{n}+, x_{\alpha}\left(t_{n}+\right)-\right)\right) \\
= & \sum_{x_{\alpha}\left(t_{n}\right) \in I\left(t_{n}\right)}\left(v^{\nu}\left(t_{n}-, x_{\alpha}\left(t_{n}\right)+\right)-v^{\nu}\left(t_{n}-, x_{\alpha}\left(t_{n}\right)-\right)\right)+\Delta t^{\nu}\left(g_{\delta}^{\nu}\left(t_{n}-, x_{\alpha}\left(t_{n}\right)+\right)-g_{\delta}^{\nu}\left(t_{n}-, x_{\alpha}\left(t_{n}\right)-\right)\right) \\
= & \widetilde{M}^{\nu}\left(t_{n}-\right)+\sum_{x_{\alpha}\left(t_{n}\right) \in I\left(t_{n}\right)} \Delta t^{\nu}\left(g_{\delta}^{\nu}\left(t_{n}-, x_{\alpha}\left(t_{n}\right)+\right)-g_{\delta}^{\nu}\left(t_{n}-, x_{\alpha}\left(t_{n}\right)-\right)\right)
\end{aligned}
$$

where we used that if there is a 1-wave in $x_{\alpha}\left(t_{n}-\right)$, then there is at least one 1-wave in $x_{\alpha}\left(t_{n}+\right)$. For each $\nu$, we define $N^{\nu}$ such that $T \in\left[N^{\nu} \Delta t^{\nu},\left(N^{\nu}+1\right) \Delta t^{\nu}\right.$. Assume now $t \in\left[\left(N_{0}-\right.\right.$ 1) $\Delta t^{\nu}, N_{0} \Delta t^{\nu}$. Since $\widetilde{M}^{\nu}$ is constant outside of splitting times, we have:

$$
\begin{aligned}
\widetilde{M}^{\nu}(T) & =\widetilde{M}^{\nu}(t)+\sum_{n=N_{0}}^{N^{\nu}} \Delta \widetilde{M}\left(t_{n}\right) \\
& =\widetilde{M}^{\nu}(t)+\sum_{n=N_{0}}^{N^{\nu}} \sum_{x_{\alpha}\left(t_{n}\right) \in I\left(t_{n}\right)} \Delta t^{\nu}\left(g_{\delta}^{\nu}\left(t_{n}-, x_{\alpha}\left(t_{n}\right)+\right)-g_{\delta}^{\nu}\left(t_{n}-, x_{\alpha}\left(t_{n}\right)-\right)\right)
\end{aligned}
$$

Therefore we have:

$$
M^{\nu}(t) \geqslant \frac{\widetilde{M}^{\nu}(t)}{c_{M}}=\frac{1}{c_{M}}\left(\widetilde{M}^{\nu}(T)-\sum_{n=N_{0}}^{N^{\nu}} \sum_{x_{\alpha}\left(t_{n}\right) \in I\left(t_{n}\right)} \Delta t^{\nu}\left(g_{\delta}^{\nu}\left(t_{n}-, x_{\alpha}\left(t_{n}\right)+\right)-g_{\delta}^{\nu}\left(t_{n}-, x_{\alpha}\left(t_{n}\right)-\right)\right)\right)
$$




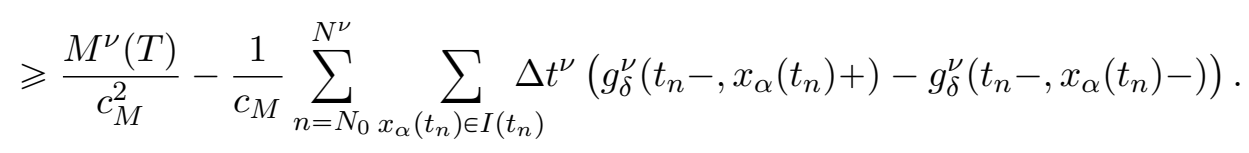

Then from 4.7 we recover

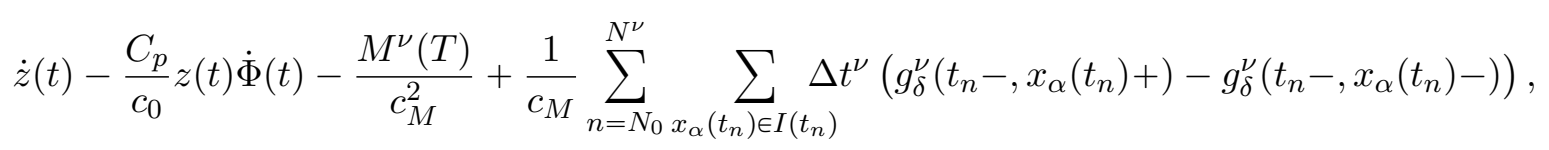

which can be rewritten as

$$
\begin{aligned}
\dot{z}(t)-\frac{C_{p}}{c_{0}} z(t) \dot{\Phi}(t)-\frac{M^{\nu}(T)}{c_{M}^{2}} & \geqslant-\frac{1}{c_{M}} \sum_{n=N_{0}}^{N^{\nu}} \sum_{x_{\alpha}\left(t_{n}\right) \in I\left(t_{n}\right)} \Delta t^{\nu}\left(g_{\delta}^{\nu}\left(t_{n}-, x_{\alpha}\left(t_{n}\right)+\right)-g_{\delta}^{\nu}\left(t_{n}-, x_{\alpha}\left(t_{n}\right)-\right)\right) \\
& \geqslant-\frac{1}{c_{M}} \sum_{n=1}^{N^{\nu}} \sum_{x_{\alpha}\left(t_{n}\right) \in I\left(t_{n}\right)} \Delta t^{\nu}\left|\left(g_{\delta}^{\nu}\left(t_{n}-, x_{\alpha}\left(t_{n}\right)+\right)-g_{\delta}^{\nu}\left(t_{n}-, x_{\alpha}\left(t_{n}\right)-\right)\right)\right| \\
& \geqslant-\frac{1}{c_{M}} \sum_{n=1}^{N^{\nu}} \Delta t^{\nu} T V\left(g_{\delta}^{\nu}\left(t_{n}-, \cdot\right) ; I\left(t_{n}\right)\right) .
\end{aligned}
$$

Hence the solution of 4.7 satisfies:

$$
\begin{aligned}
z(T) & \geqslant e^{\int_{0}^{T} \frac{C_{p}}{c_{0}} \dot{\Phi}(t) d t}\left[z(0)+\left(\frac{M^{\nu}(T)}{c_{M}^{2}}-\frac{1}{c_{M}} \sum_{n=1}^{N^{\nu}} \Delta t^{\nu} T V\left(g_{\delta}^{\nu}\left(t_{n}-, \cdot\right) ; I\left(t_{n}\right)\right)\right) \int_{0}^{T} e^{-\int_{0}^{t} \frac{C_{p}}{c_{0}} \dot{\Phi}(s) d s} d t\right] \\
& \geqslant e^{\int_{0}^{T} \frac{C_{p}}{c_{0}} \dot{\Phi}(t) d t}\left[\left(\frac{M^{\nu}(T)}{c_{M}^{2}}-\frac{1}{c_{M}} \sum_{n=1}^{N^{\nu}} \Delta t^{\nu} T V\left(g_{\delta}^{\nu}\left(t_{n}-, \cdot\right) ; I\left(t_{n}\right)\right)\right) \int_{0}^{T} e^{-\int_{0}^{t} \frac{C_{p}}{c_{0}} \dot{\Phi}(s) d s} d t\right],
\end{aligned}
$$

which gives

$$
\begin{aligned}
M^{\nu}(T) & \leqslant \frac{c_{M}^{2} z(T) e^{-\int_{0}^{T} \frac{C_{p}}{c_{0}} \dot{\Phi}(t) d t}}{\int_{0}^{T} e^{-\int_{0}^{t} \frac{C_{p}}{c_{0}} \dot{\Phi}(s) d s} d t}+c_{M} \sum_{n=1}^{N^{\nu}} \Delta t^{\nu} T V\left(g_{\delta}^{\nu}\left(t_{n}-, \cdot\right) ; I\left(t_{n}\right)\right) \\
& \leqslant \frac{c_{M}^{2} z(T)}{T} e^{-\int_{0}^{T} \frac{C_{p}}{c_{0}} \dot{\Phi}(t) d t} d t+c_{M} \sum_{n=1}^{N^{\nu}} \Delta t^{\nu} T V\left(g_{\delta}^{\nu}\left(t_{n}-, \cdot\right) ; I\left(t_{n}\right)\right) .
\end{aligned}
$$

By the same process as above, we can estimate the contribution given by $\Phi$ as follows:

$$
\begin{aligned}
\int_{0}^{T} \dot{\Phi}(t) d t & =\Phi(T)-\sum_{n=1}^{N^{\nu}}(\Phi(n \Delta t+)-\Phi(n \Delta t-))-\Phi(0) \\
& \geqslant \Phi(T)-\Phi(0)-\sum_{n=1}^{N^{\nu}}|\Delta \Phi(n \Delta t)| \\
& \geqslant \Phi(T)-\Phi(0)-\sum_{n=1}^{N^{\nu}}\left|\Delta K^{\nu}(n \Delta t)\right| \\
& \geqslant \Phi(T)-\Phi(0)-\sum_{n=1}^{N^{\nu}} \Delta t^{\nu} T V\left(g_{\delta}^{\nu}\left(t_{n}-, \cdot\right) ; I\left(t_{n}\right)\right)
\end{aligned}
$$


since $\phi$ is time-continuous, and we obtain

$$
\begin{aligned}
M^{\nu}(T) & \leqslant \frac{c_{M}^{2} z(T)}{T} e^{-\int_{0}^{T} \frac{C_{p}}{c_{0}} \dot{\Phi}(t) d t} d t+c_{M} \sum_{n=1}^{N^{\nu}} \Delta t^{\nu} T V\left(g_{\delta}^{\nu}\left(t_{n}-, \cdot\right) ; I\left(t_{n}\right)\right) \\
& \leqslant \frac{c_{M}^{2} z(T)}{T} e^{\frac{C_{p}}{c_{0}}\left(\Phi(0)-\Phi(T)+\sum_{n=1}^{N^{\nu}} \Delta t^{\nu} T V\left(g_{\delta}^{\nu}\left(t_{n}-, \cdot\right) ; I\left(t_{n}\right)\right)\right)}+c_{M} \sum_{n=1}^{N^{\nu}} \Delta t^{\nu} T V\left(g_{\delta}^{\nu}\left(t_{n}-, \cdot\right) ; I\left(t_{n}\right)\right) .
\end{aligned}
$$

This estimate remains valid for any finite number $l$ of disjoints intervals $\left.\left.I_{i}=\right] a_{i}, b_{i}\right]$. We call $M_{i}^{\nu}(T)$ the sum of strength of of 1 -waves in $W^{\nu}(T,$.$\left.\left.) contained in \right] a_{i}, b_{i}\right]$ and write $T V\left(g_{\delta}^{\nu}(t, \cdot) ; I_{i}(t)\right)$ the total variation associated to the interval $\left.] a_{i}(t), b_{i}(t)\right]$ :

$$
M_{i}(T)=\sum_{\left.\left.k_{\alpha}=1, x_{\alpha} \in\right] a_{i}, b_{i}\right]} \sigma_{\alpha}
$$

We now consider a given open-interval $] a, b[$. We assume that the front tracking approximation $U^{\nu}$ initially contains $N$ shocks emanating from the initial datum. We can now define half-open disjoint intervals $\left.\left.I_{i}=\right] a_{i}, b_{i}\right], 1 \leqslant i \leqslant N+1$, such that:

- every 1-rarefaction front in $W^{\nu}$ at $T$ lying in $] a, b\left[\right.$ is contained in an interval $I_{i}$.

- each interval $I_{i}$ can only contain shocks originating from the integration of the splitting term at a time $t_{n}$, and cannot contain shocks originating from the initial datum.

The measure of positive 1 -waves in $W^{\nu}(T, \cdot)$ is then

$$
\mu_{T}^{1+}(] a, b[)=\sum_{i=1}^{l} M_{i}(T) .
$$

We have the following bound:

$$
\sum_{i=1}^{l} T V_{i}\left(g_{\delta}^{\nu}\left(t_{n}-, \cdot\right) ; I_{i}\left(t_{n}\right)\right) \leqslant T V\left(g_{\delta}^{\nu}\left(t_{n}-, \cdot\right) ;\right] a, b[) .
$$

By definition of $T g_{\delta}^{\nu}(t, \cdot)$, we have

$$
\begin{aligned}
T V\left(g_{\delta}^{\nu}(t, \cdot) ;\right] a, b[) & \leqslant \frac{1}{\delta} T V\left(\tilde{V} \circ \tilde{p}^{-1}\left(w^{\nu}-v^{\nu}\right)(t, \cdot) ;\right] a, b[)+T V\left(v^{\nu}(t, \cdot) ;\right] a, b[) \\
& \leqslant \frac{C_{V p}}{\delta}\left(T V\left(w^{\nu}(t, \cdot) ;\right] a, b[)+T V\left(v^{\nu}(t, \cdot) ;\right] a, b[)\right) \\
& \leqslant \frac{C_{V p}}{\delta}\left(T V\left(w_{0}^{\nu}\right)+T V\left(v_{0}^{\nu}\right)\right) \\
& \leqslant \frac{C_{V p}}{\delta}\left(T V\left(w_{0}\right)+T V\left(v_{0}\right)\right)
\end{aligned}
$$

where we used the Lipschitz-continuity of $\tilde{V} \circ \tilde{p}^{-1}$ on the domain $\mathbf{E}$ and the result of Lemma 2.2 . Finally we get

$$
\begin{aligned}
\mu_{T}^{1+}(] a, b[) & \leqslant \sum_{i=1}^{l} \frac{c_{M}^{2}\left(b_{i}-a_{i}\right)}{T} e^{\frac{C_{p}}{c_{0}}\left(\Phi_{i}(0)-\Phi_{i}(T)+T \frac{C_{V p}}{\delta}\left(T V\left(w_{0}\right)+T V\left(v_{0}\right)\right)\right)}+c_{M} T \frac{C_{V p}}{\delta}\left(T V\left(w_{0}\right)+T V\left(v_{0}\right)\right) \\
& \leqslant c_{M}^{2} \frac{(b-a)}{T} e^{\frac{C_{p}}{c_{0}}\left(\Phi(0)+T \frac{C_{V p}}{\delta}\left(T V\left(w_{0}\right)+T V\left(v_{0}\right)\right)\right)}+c_{M} T \frac{C_{V p}}{\delta}\left(T V\left(w_{0}\right)+T V\left(v_{0}\right)\right)
\end{aligned}
$$




$$
\leqslant c_{M}^{2} \frac{b-a}{T} e^{\frac{C_{p}}{c_{0}}\left(T V\left(w_{0}\right)+T \frac{C_{V p}}{\delta}\left(T V\left(w_{0}\right)+T V\left(v_{0}\right)\right)\right)}+c_{M} C_{V p} \frac{T}{\delta}\left(T V\left(w_{0}\right)+T V\left(v_{0}\right)\right)
$$

proving 4.2 with $C=\max \left\{c_{M}^{2} e^{\frac{C_{p}}{c_{0}} T V\left(w_{0}\right)}, c_{M} C_{V p}, \frac{C_{p}}{c_{0}}, \frac{C_{p} C_{V p}}{c_{0}}\right\}$.

Remark. Unfortunately, the lower semicontinuity of the total variation does not allow to pass to the limit as $\nu \rightarrow \infty$ in the term $T V\left(g_{\delta}^{\nu}\left(t_{n}-, \cdot\right) ;\right] a, b[)$ to recover a sharper estimate depending on the time integral of the total variation of the relaxation term.

\section{Convergence of the relaxed ARZ system towards LWR equa- tion}

We follow the methodology of 2]. For each $\delta>0$, we constructed a sequence of approximate WFT solutions whose limit solves $(1.9)$ in the weak sense. We recall that, under the assumptions of Lemma 2.3 , for any $a<b$ and $0 \leqslant s \leqslant t$ they satisfy estimates (2.6) and 2.7):

$$
\begin{aligned}
& \int_{a}^{b}\left|\tau^{\nu}(t, X)-\tau^{\nu}(s, X)\right| d X \leqslant C_{M}(t-s) \\
& \int_{a}^{b}\left|w^{\nu}(t, X)-w^{\nu}(s, X)\right| d X \leqslant\left(C_{M}+L_{\delta}\right)(t-s+\Delta t) .
\end{aligned}
$$

To pass to the limit as $\delta \rightarrow 0$, we need a stronger estimate on $L_{\delta}$.

Lemma 5.1. Assume that the sub-characteristic condition (1.11) is satisfied. Under the same assumptions of Lemma 2.3, we have the following estimate:

$$
L_{\delta} \leqslant \frac{2}{\delta} e^{-\frac{s}{\delta}} \int_{a}^{b}\left|\tilde{V}\left(\tau_{0}(X)\right)-v_{0}(X)\right| d X
$$

Proof. Let $\delta>0$ and $\nu$ be given. For each $k \geqslant 1$, we define:

$$
g_{k}^{ \pm}=\int_{a}^{b}\left|\tilde{V}\left(\tau^{\nu}\left(k \Delta t^{\nu} \pm, X\right)\right)-v^{\nu}\left(k \Delta t^{\nu} \pm, X\right)\right| d X
$$

We develop

$$
\begin{aligned}
g_{k}^{+} & =\int_{a}^{b}\left|\tilde{V}\left(\tau^{\nu}\left(k \Delta t^{\nu}+, X\right)\right)-v^{\nu}\left(k \Delta t^{\nu}+, X\right)\right| d X \\
& =\int_{a}^{b}\left|\tilde{V}\left(\tau^{\nu}\left(k \Delta t^{\nu}-, X\right)\right)-\left(v^{\nu}\left(k \Delta t^{\nu}-, X\right)+\frac{\Delta t^{\nu}}{\delta}\left(\tilde{V}\left(\tau^{\nu}\left(k \Delta t^{\nu}-, X\right)\right)-v^{\nu}\left(k \Delta t^{\nu}-, X\right)\right)\right)\right| d X \\
& =\left(1-\frac{\Delta t^{\nu}}{\delta}\right) g_{k}^{-}
\end{aligned}
$$

Therefore we get

$$
\begin{aligned}
& g_{k}^{-}-g_{k-1}^{+}= \\
= & \int_{a}^{b}\left|\tilde{V}\left(\tau^{\nu}\left(k \Delta t^{\nu}-, X\right)\right)-v^{\nu}\left(k \Delta t^{\nu}-, X\right)\right|-\left|\tilde{V}\left(\tau^{\nu}\left((k-1) \Delta t^{\nu}+, X\right)\right)-v^{\nu}\left((k-1) \Delta t^{\nu}+, X\right)\right| d X
\end{aligned}
$$




$$
\begin{aligned}
\leqslant & \int_{a}^{b}\left|\left(\tilde{V}\left(\tau^{\nu}\left(k \Delta t^{\nu}-, X\right)\right)-v^{\nu}\left(k \Delta t^{\nu}-, X\right)\right)-\left(\tilde{V}\left(\tau^{\nu}\left((k-1) \Delta t^{\nu}+, X\right)\right)-v^{\nu}\left((k-1) \Delta t^{\nu}+, X\right)\right)\right| d X \\
\leqslant & \int_{a}^{b} \mid\left(\tilde{V}\left(\tau^{\nu}\left(k \Delta t^{\nu}-, X\right)\right)-\tilde{V}\left(\tau^{\nu}\left((k-1) \Delta t^{\nu}+, X\right)\right)\right)-\left(w^{\nu}\left(k \Delta t^{\nu}-, X\right)-w^{\nu}\left((k-1) \Delta t^{\nu}+, X\right)\right) \\
& +\left(\tilde{p}\left(\tau^{\nu}\left(k \Delta t^{\nu}-, X\right)\right)-\tilde{p}\left(\tau^{\nu}\left((k-1) \Delta t^{\nu}+, X\right)\right)\right) \mid d X \\
\leqslant & \sup _{U^{\nu} \in \mathcal{D}(M)}\left(\left|\tilde{V}^{\prime}\left(\tau^{\nu}\right)\right|+\left|\tilde{p}^{\prime}\left(\tau^{\nu}\right)\right|\right) C_{M} \Delta t^{\nu} \\
\leqslant & K_{1} \Delta t^{\nu}
\end{aligned}
$$

where $K_{1}:=\sup _{U^{\nu} \in \mathcal{D}(M)}\left(\left|\tilde{V}^{\prime}\left(\tau^{\nu}\right)\right|+\left|\tilde{p}^{\prime}\left(\tau^{\nu}\right)\right|\right) C_{M}$. Combining 5.2 and 5.3 we obtain:

$$
\begin{aligned}
g_{k}^{-} & \leqslant K_{1} \Delta t^{\nu} \sum_{i=0}^{k-1}\left(1-\frac{\Delta t^{\nu}}{\delta}\right)^{i}+\left(1-\frac{\Delta t^{\nu}}{\delta}\right)^{k-1} g_{0}^{+} \\
& \leqslant K_{1} \delta+\left(1-\frac{\Delta t^{\nu}}{\delta}\right)^{k-1} g_{0}^{+} .
\end{aligned}
$$

As in the proof of Lemma 2.3, we write:

$$
\begin{aligned}
& \sum_{i=k}^{k+N^{\nu}} \int_{a}^{b}\left|w^{\nu}\left(i \Delta t^{\nu}+, X\right)-w^{\nu}\left(i \Delta t^{\nu}-, X\right)\right| d X= \\
& \quad=\frac{\Delta t^{\nu}}{\delta} \sum_{i=k}^{k+N^{\nu}} g_{i}^{-} \\
& \quad \leqslant \frac{\Delta t^{\nu}}{\delta} \sum_{i=k}^{k+N^{\nu}}\left[K_{1} \delta+\left(1-\frac{\Delta t^{\nu}}{\delta}\right)^{i-1} g_{0}^{+}\right] \\
& \quad \leqslant K_{1}\left(t-s+\Delta t^{\nu}\right)+g_{0}^{+}\left(1-\frac{\Delta t^{\nu}}{\delta}\right)^{k-1}\left[1-\left(1-\frac{\Delta t^{\nu}}{\delta}\right)^{N^{\nu}+1}\right]
\end{aligned}
$$

Taking $\Delta t^{\nu} \leqslant \frac{\delta}{2}$, we have $2\left(1-\frac{\Delta t^{\nu}}{\delta}\right) \geqslant 1$ and

$$
\begin{aligned}
\sum_{i=k}^{k+N^{\nu}} \int_{a}^{b}\left|w^{\nu}\left(i \Delta t^{\nu}+, X\right)-w^{\nu}\left(i \Delta t^{\nu}-, X\right)\right| d X & \leqslant K_{1}\left(t-s+\Delta t^{\nu}\right)+2 g_{0}^{+}\left(1-\frac{\Delta t^{\nu}}{\delta}\right)^{k}(N+1) \frac{\Delta t^{\nu}}{\delta} \\
& \leqslant\left(t-s+\Delta t^{\nu}\right)\left[K_{1}+2 \frac{g_{0}^{+}}{\delta} e^{-\frac{s}{\delta}}\right] .
\end{aligned}
$$

Then the result holds with another constant $C_{M}$ large enough.

We can now present the main result of this paper.

Theorem 5.2. Let $U_{0}=\left(\tau_{0}, w_{0}\right) \in \mathcal{D}(M)$ for some $M>0$, and denote by $\bar{U}=(\bar{\tau}, \bar{w})$ the limit as $\delta \rightarrow 0$ of a subsequence of weak entropy solutions $U^{\delta}=\left(\tau^{\delta}, w^{\delta}\right)$ of 1.9$)$, 1.10), obtained as limit of the proposed Wave-Front-Tracking approximations. Then $\bar{w}=V(\bar{\tau})+\tilde{p}(\bar{\tau})$ and $\bar{\tau}$ is a weak solution of the scalar Cauchy problem:

$$
\left\{\begin{array}{l}
\partial_{t} \tau-\partial_{X} \tilde{V}(\tau)=0, \\
\tau(0, \cdot)=\tau_{0}(\cdot),
\end{array} \quad X \in \mathbb{R}, t>0 .\right.
$$


Proof. First we remind that the constant $C_{M}$ in $(2.6)$ and $(2.7)$ does not depend on $\delta$. In addition, thanks to (5.1), the constant $L_{\delta}$ can be bounded uniformly as $\delta \rightarrow 0$ on any set $\left[1 / n, \infty\left[\times[-n, n]\right.\right.$ for $n \in \mathbb{N}$. If $\frac{1}{n} \leqslant s \leqslant t$ then, for a given constant $C$ only depending on the initial datum, there holds

$$
L_{\delta} \leqslant \frac{C}{\delta}(b-a) e^{-\frac{1}{n \delta}}
$$

For $\mathrm{n}$ fixed, the right-hand side goes to zero with $\delta$.

By Helly's theorem, there exists a subsequence $\delta_{k} \rightarrow 0$, such that the sequence $\left(\tau^{\delta_{k}}\right)$ converges to a function $\bar{\tau}$ in $\mathbb{L}_{l o c}^{1}\left(\left[0,+\infty[\times \mathbb{R})\right.\right.$. In addition we have by construction $\bar{\tau}(0, \cdot)=\tau_{0}(\cdot)$ and the limit also satisfies the Lipschitz inequality.

For $\bar{w}$ we can reason on the set $\left[1,+\infty\left[\times[-1,1]\right.\right.$. We can extract a subsequence $\delta_{k}^{1}$ from $\delta_{k}$ such that $w^{\delta_{k}^{1}}$ converges to a function $\bar{w}$ in $\mathbb{L}_{l o c}^{1}([1,+\infty[\times[-1,1])$. Passing to the limit in 3.1 we obtain

$$
\bar{w}(t, \cdot)=\tilde{V}(\bar{\tau}(t, \cdot))+\tilde{p}(\bar{\tau}(t, \cdot))
$$

on the set $\left[1,+\infty\left[\times[-1,1]\right.\right.$, and then $\bar{\tau}_{t}-\tilde{V}(\bar{\tau})_{x}=0$.

Similarly, for any $n \in \mathbb{N}$, we can extract a subsequence $\delta_{k}^{n}$ of $\delta_{k}^{n-1}$ such that $w_{k}^{\delta_{k}^{n}}$ converges to $\bar{w}$ in $\mathbb{L}_{l o c}^{1}\left(\left[1 / n,+\infty[\times[-n, n])\right.\right.$. For any $\frac{1}{n} \leqslant s \leqslant t,(5.5)$ combined with 2.7 provides:

$$
\int_{-n}^{n}|\bar{w}(t, X)-\bar{w}(s, X)| d X \leqslant C_{M}|t-s| .
$$

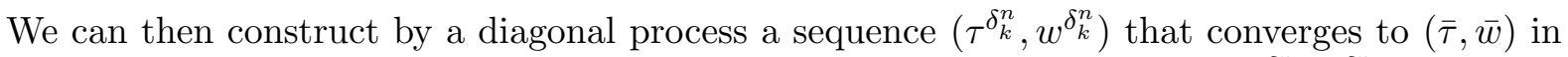
$\mathbb{L}_{l o c}^{1}(] 0,+\infty[\times \mathbb{R})$. For any compact subset of $\left[0,+\infty\left[\times \mathbb{R}\right.\right.$, we know that $\left(\tau^{\delta_{k}^{n}}, w^{\delta_{k}^{n}}\right)$ converges to $(\bar{\tau}, \bar{w})$ in $\mathbb{L}_{l o c}^{1}([1 / n,+\infty[\times[-n, n])$, and it is bounded by construction. Therefore, we get convergence in $\mathbb{L}_{\text {loc }}^{1}([0,+\infty[\times \mathbb{R})$.

Inequality (5.6) holds for any $0<s<t$ and any $n \in \mathbb{N}$, we can thus pass to the limit when $t \rightarrow 0$, and define:

$$
\bar{w}(0, \cdot)=\lim _{t \rightarrow 0} \bar{w}(t, \cdot)=\lim _{t \rightarrow 0} \tilde{V}(\bar{\tau}(t, \cdot))+\tilde{p}(\bar{\tau}(t, \cdot))=\tilde{V}\left(\tau_{0}(\cdot)\right)+\tilde{p}\left(\tau_{0}(\cdot)\right) .
$$

Then $\bar{\tau}$ is a weak solution of (5.4), which corresponds to the usual LWR model.

Remark. Proving that the solutions of the ARZ system with relaxation 1.9 converge to the (unique) entropy weak solution of (5.4) is still an open problem, see [27, Section 5] and [2, Section 4] for related discussions. The mild decay estimate 4.2 does not give useful information for $\delta \rightarrow 0$.

\section{Acknowledgements}

The authors thank Debora Amadori and Graziano Guerra for insightful discussions.

\section{Appendix A $T V$ bounds: Failure of Godunov scheme}

The present work was motivated by the lack of complete convergence results of the relaxed ARZ model (1.4) to the equilibrium LWR equation

$$
\partial_{t} \rho+\partial_{x} V(\rho)=0
$$


Following [4, 23, we made the choice of working in Lagrangian coordinates, which greatly simplifies computations. The same procedure could be carried out for the original problem in Eulerian coordinates. Besides, as mentioned in the introduction, the choice of the WFT scheme for constructing approximate solutions was motivated by the lack of $T V$ bounds on classical Godunov approximations. This was already pointed out in [37] for system (1.4), but remains valid in Lagrangian coordinates. Indeed, Godunov scheme for the homogeneous step (2.3) reads (see [4, Eq. (4.8)])

$$
\begin{aligned}
\tau_{i}^{n+1} & =\tau_{i}^{n}+\frac{\Delta t}{\Delta X}\left(v_{i+1}^{n}-v_{i}^{n}\right), \\
w_{i}^{n+1} & =w_{i}^{n},
\end{aligned}
$$

see also Figure 2, Note that, in general, the solution of the Riemann problem with initial data $U_{i}^{n}, U_{i+1}^{n}$, accounts for a wave of the first family traveling with negative speed and a stationary contact discontinuity.

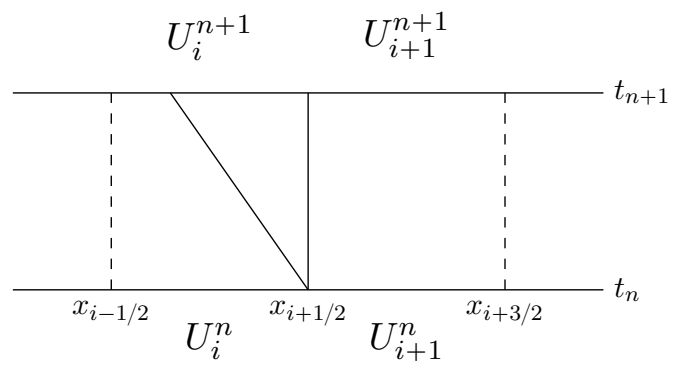

Figure 2: Notations used for Godunov scheme, with $U_{i}^{n}=\left(\tau_{i}^{n}, w_{i}^{n}\right)$.

By construction, and relying on the fact that $\tau$ is a conserved variable, we have that $\tau_{i}^{n+1} \in I\left(\tau_{i}^{n}, \tau_{i+1}^{n}\right)$, with the notation $I(a, b):=[\min \{a, b\}, \max \{a, b\}]$. Therefore $\tilde{p}\left(\tau_{i}^{n+1}\right) \in$ $I\left(\tilde{p}\left(\tau_{i}^{n}\right), \tilde{p}\left(\tau_{i+1}^{n}\right)\right)$, since $\tilde{p}$ is monotone. This gives

$$
v_{i}^{n+1} \in I\left(w_{i}^{n}-\tilde{p}\left(\tau_{i}^{n}\right), w_{i}^{n}-\tilde{p}\left(\tau_{i+1}^{n}\right)\right)=I\left(v_{i}^{n}, v_{i+1}^{n}+\left(w_{i}^{n}-w_{i+1}^{n}\right)\right) \neq I\left(v_{i}^{n}, v_{i+1}^{n}\right),
$$

since $w_{i}^{n}-w_{i+1}^{n} \neq 0$ in general. In particular, we may have $v_{i}^{n+1} \notin I\left(v_{i}^{n}, v_{i+1}^{n}\right)$. This may generate spurious oscillations around contact discontinuities, see for example [13].

\section{References}

[1] D. Amadori and A. Corli. Global existence of BV solutions and relaxation limit for a model of multiphase reactive flow. Nonlinear Anal., 72(5):2527-2541, 2010.

[2] D. Amadori and G. Guerra. Global BV solutions and relaxation limit for a system of conservation laws. Proc. Roy. Soc. Edinburgh Sect. A, 131(1):1-26, 2001.

[3] F. Ancona and P. Goatin. Uniqueness and stability of $L^{\infty}$ solutions for Temple class systems with boundary and properties of the attainable sets. SIAM J. Math. Anal., 34(1):28-63, 2002. 
[4] A. Aw, A. Klar, T. Materne, and M. Rascle. Derivation of continuum traffic flow models from microscopic follow-the-leader models. SIAM J. Appl. Math., 63(1):259-278, 2002.

[5] A. Aw and M. Rascle. Resurrection of "second order" models of traffic flow. SIAM J. Appl. Math., 60(3):916-938, 2000.

[6] P. Bagnerini and M. Rascle. A multiclass homogenized hyperbolic model of traffic flow. SIAM J. Math. Anal., 35(4):949-973, 2003.

[7] P. Baiti and A. Bressan. The semigroup generated by a Temple class system with large data. Differential Integral Equations, 10(3):401-418, 1997.

[8] A. Bressan. Hyperbolic systems of conservation laws, volume 20 of Oxford Lecture Series in Mathematics and its Applications. Oxford University Press, Oxford, 2000.

[9] A. Bressan and R. M. Colombo. Decay of positive waves in nonlinear systems of conservation laws. Ann. Scuola Norm. Sup. Pisa Cl. Sci. (4), 26(1):133-160, 1998.

[10] A. Bressan and P. Goatin. Oleinik type estimates and uniqueness for $n \times n$ conservation laws. J. Differential Equations, 156(1):26-49, 1999.

[11] A. Bressan and P. Goatin. Stability of $L^{\infty}$ solutions of Temple class systems. Differential Integral Equations, 13(10-12):1503-1528, 2000.

[12] A. Bressan and T. Yang. A sharp decay estimate for positive nonlinear waves. SIAM J. Math. Anal., 36(2):659-677, 2004.

[13] C. Chalons and P. Goatin. Godunov scheme and sampling technique for computing phase transitions in traffic flow modeling. Interfaces Free Bound., 10(2):197-221, 2008.

[14] G. Q. Chen, C. D. Levermore, and T.-P. Liu. Hyperbolic conservation laws with stiff relaxation terms and entropy. Comm. Pure Appl. Math., 47(6):787-830, 1994.

[15] G. Q. Chen and T.-P. Liu. Zero relaxation and dissipation limits for hyperbolic conservation laws. Comm. Pure Appl. Math., 46(5):755-781, 1993.

[16] C. Christoforou and K. Trivisa. Sharp decay estimates for hyperbolic balance laws. J. Differential Equations, 247(2):401-423, 2009.

[17] C. Christoforou and K. Trivisa. Decay of positive waves of hyperbolic balance laws. Acta Math. Sci. Ser. B Engl. Ed., 32(1):352-366, 2012.

[18] R. Courant and K. O. Friedrichs. Supersonic Flow and Shock Waves. Interscience Publishers, Inc., New York, N. Y., 1948.

[19] G. Crasta and B. Piccoli. Viscosity solutions and uniqueness for systems of inhomogeneous balance laws. Discrete Contin. Dynam. Systems, 3(4):477-502, 1997.

[20] C. M. Dafermos. Hyperbolic conservation laws in continuum physics, volume 325 of Grundlehren der Mathematischen Wissenschaften [Fundamental Principles of Mathematical Sciences]. Springer-Verlag, Berlin, 2000.

[21] C. F. Daganzo. Requiem for second-order fluid approximations of traffic flow. Transportation Research Part B: Methodological, 29(4):277-286, 1995. 
[22] P. Goatin and L. Gosse. Decay of positive waves for $n \times n$ hyperbolic systems of balance laws. Proc. Amer. Math. Soc., 132(6):1627-1637, 2004.

[23] J. M. Greenberg. Extensions and amplifications of a traffic model of Aw and Rascle. SIAM J. Appl. Math., 62(3):729-745, 2001/02.

[24] D. Hoff. Invariant regions for systems of conservation laws. Trans. Amer. Math. Soc., 289(2):591-610, 1985.

[25] H. Holden and N. H. Risebro. Front tracking for hyperbolic conservation laws, volume 152 of Applied Mathematical Sciences. Springer-Verlag, New York, 2002.

[26] H. K. Jenssen and C. Sinestrari. On the spreading of characteristics for non-convex conservation laws. Proc. Roy. Soc. Edinburgh Sect. A, 131(4):909-925, 2001.

[27] C. Lattanzio and P. Marcati. The zero relaxation limit for the hydrodynamic Whitham traffic flow model. J. Differential Equations, 141(1):150-178, 1997.

[28] T. Li. Global solutions and zero relaxation limit for a traffic flow model. SIAM J. Appl. Math., 61(3):1042-1061, 2000.

[29] T. Li. $L^{1}$ stability of conservation laws for a traffic flow model. Electron. J. Differential Equations, pages No. 14, 18, 2001.

[30] T. Li. Well-posedness theory of an inhomogeneous traffic flow model. Discrete Contin. Dyn. Syst. Ser. B, 2(3):401-414, 2002.

[31] T. Li. Global solutions of nonconcave hyperbolic conservation laws with relaxation arising from traffic flow. J. Differential Equations, 190(1):131-149, 2003.

[32] T. Li and H. Liu. Critical thresholds in a relaxation model for traffic flows. Indiana Univ. Math. J., 57(3):1409-1430, 2008.

[33] M. J. Lighthill and G. B. Whitham. On kinematic waves. II. A theory of traffic flow on long crowded roads. Proc. Roy. Soc. London. Ser. A., 229:317-345, 1955.

[34] O. A. Oleĭ nik. Discontinuous solutions of non-linear differential equations. Uspehi Mat. Nauk (N.S.), 12(3(75)):3-73, 1957.

[35] O. A. Oleŭ nik. Discontinuous solutions of non-linear differential equations. Amer. Math. Soc. Transl. (2), 26:95-172, 1963.

[36] H. J. Payne. Models of freeway traffic and control. Mathematical models of public systems, 1971.

[37] M. Rascle. An improved macroscopic model of traffic flow: derivation and links with the Lighthill-Whitham model. Math. Comput. Modelling, 35(5-6):581-590, 2002.

[38] P. I. Richards. Shock waves on the highway. Operations Res., 4:42-51, 1956.

[39] D. Serre. Systèmes de lois de conservation II. structures géométriques, oscillation et problèmes mixtes. 1996.

[40] B. Temple. Systems of conservation laws with invariant submanifolds. Trans. Amer. Math. Soc., 280(2):781-795, 1983. 
[41] D. H. Wagner. Equivalence of the Euler and Lagrangian equations of gas dynamics for weak solutions. J. Differential Equations, 68(1):118-136, 1987.

[42] G. B. Whitham. Linear and nonlinear waves. pages xvi+636, 1974. Pure and Applied Mathematics.

[43] H. M. Zhang. A non-equilibrium traffic model devoid of gas-like behavior. Transportation Research Part B: Methodological, 36(3):275-290, 2002. 